\title{
Ensemble Forecasts and the Properties of Flow-Dependent Analysis-Error Covariance Singular Vectors
}

\author{
Thomas M. HAMILL \\ University of Colorado, and NOAA-CIRES Climate Diagnostics Center, Boulder, Colorado \\ CHRIS SNYDER \\ National Center for Atmospheric Research, * Boulder, Colorado \\ JEFFREY S. WHITAKER \\ NOAA-CIRES Climate Diagnostics Center, Boulder, Colorado
}

(Manuscript received 9 May 2002, in final form 21 January 2003)

\begin{abstract}
Approximations to flow-dependent analysis-error covariance singular vectors (AEC SVs) were calculated in a dry, T31 L15 primitive-equation global model. Sets of 400-member ensembles of analyses were generated by an ensemble-based data assimilation system. A sparse network of simulated rawinsonde observations were assimilated, and a perfect model was assumed. Ensembles of 48-h forecasts were also generated from these analyses. The structure of evolved singular vectors was determined by finding the linear combination of the forecast ensemble members that resulted in the largest forecast-error variance, here measured in a total-energy norm north of $20^{\circ} \mathrm{N}$ latitude. The same linear combination of analyses specifies the initial-time structure that should evolve to the forecast singular vector under assumptions of linearity of error growth.

The structures of these AEC SVs are important because they represent the analysis-error structures associated with the largest forecast errors. If singular vectors using other initial norms have very different structures, this indicates that these structures may be statistically unlikely to occur. The European Centre for Medium-Range Weather Forecasts currently uses singular vectors using an initial total-energy norm ["total-energy singular vectors" or (TE SVs)] to generate perturbations to initialize their ensemble forecasts. Approximate TE SVs were also calculated by drawing an initial random ensemble with perturbations that were white in total energy and applying the same approach as for AEC SVs. Comparing AEC SVs and approximate TE SVs, the AEC SVs had maximum amplitude in midlatitudes near the tropopause, both at the initial and evolved times. The AEC SVs were synoptic in scale, deep, and did not appear to be geographically localized nor tilted dramatically upshear. This contrasts with TE SVs, which started off relatively smaller in scale, were tilted upshear, and had amplitudes typically largest in the lower to midtroposphere.

The difference between AEC SVs and TE SVs suggests that operational ensemble forecasts based on TE SVs could be improved by changing the type of singular vector used to generate initial perturbations. This is particularly true for short-range ensemble forecasts, where the structure of the forecast ensemble is more closely tied to the analysis ensemble.
\end{abstract}

\section{Introduction}

Though ensemble forecasting has been operational in the United States, Europe, and Canada for nearly a decade now, no clear consensus has yet evolved on the best practical method for generating initial conditions for these ensemble forecasts. In principle, it is understood

* The National Center for Atmospheric Research is sponsored by the National Science Foundation.

Corresponding author address: Dr. Thomas M. Hamill, NOAACIRES CDC, R/CDC 1, 325 Broadway, Boulder, CO 80305-3328. E-mail: tom.hamill@noaa.gov from the Liouville equation (e.g., Ehrendorfer 1994) that the samples should be drawn from the probability distribution of analysis states, regardless of whether they should be drawn randomly or nonrandomly. However, this analysis-error probability distribution can be highly dependent on the dynamics of the day and the observation network and thus may be difficult to calculate. Analysis errors may be quite small in the region of, say, an amply observed blocking high but much larger in the region of a sparsely observed storm track. A computationally efficient method for generating sets of initial conditions that are fully consistent with flow-dependent analysiserror statistics has yet to be demonstrated with an operational weather forecast and data assimilation system. 
As a consequence, the various operational numerical weather prediction centers have embraced different approaches to generate initial conditions for their ensemble forecasts. The European Centre for Medium-Range Weather Forecasts (ECMWF) have used "singular vector" perturbations (Molteni et al. 1996); the National Centers for Environmental Prediction (NCEP) use a "breeding" method (Toth and Kalnay 1993, 1997); and at the Canadian Meteorological Centre (CMC), a "perturbed-observation" statistical interpolation approach is used (Houtekamer et al. 1996).

Understanding the relative merits of each approach has proved difficult since each forecast center uses a different forecast model, different data assimilation approaches, and even somewhat different sets of observations. Few comparisons have been performed to test the different perturbation methods with the same analysis system, forecast model, and observations. Houtekamer and Derome (1995) compared the skill of the mean forecasts from singular vector, bred, and perturbed observation ensembles using a T21 L3 quasigeostrophic model. The authors found little difference in the skill of ensemble mean forecasts produced by the three methods. Anderson $(1996,1997)$ provided some comparisons of different approaches using the three-dimensional Lorenz (1963) model. Hamill et al. (2000) provided a comparison of singular vector, bred, and perturbed observation methods in a quasigeostrophic channel model with $O\left(10^{5}\right)$ degrees of freedom under perfect-model assumptions. This comparison suggested that the perturbed observation method produced the most consistent and skillful ensembles, especially at short forecast lead times. Early on, there was a spread-skill relationship for the perturbed-observation ensemble that did not exist in the bred and singular vector ensembles.

Since all of the operational methods that are currently utilized are approximations of various degrees, some theoretical guidance on what ideally should be used would be helpful. Under assumptions of linearity of error growth and normality of errors, Ehrendorfer and Tribbia (1997) demonstrate that forecast-error covariances for a specified lead time can be predicted most efficiently using an ensemble constructed in the subspace of the leading analysis-error covariance singular vectors (AEC SVs). These are the structures that explain the greatest forecast variance and whose initial size is consistent with the analysis-error covariance statistics.

Because of the cost and difficulty of generating such AEC SVs, ECMWF has used a surrogate approach. Their ensemble of initial conditions is constructed to lie in a subspace of the leading initial-time total-energy singular vectors (TE SVs). Perturbations evolved from these initial-time singular vectors are designed to maximize forecast error variance at $48 \mathrm{~h}$ measured in a totalenergy norm, subject to the constraint that the initial size of the perturbation is fixed and again measured in a total-energy norm. The structure of evolved SVs at $48 \mathrm{~h}$ and beyond is rather similar whatever the choice of the initial norm (Barkmeijer et al. 1998, 1999), so for medium-range forecasts, TE SVs are probably a reasonable substitute for AEC SVs.

The structure of the initial-time TE SVs has been documented in a wide variety of models, with similarities more notable than the differences. From the Charney model (Farrell 1989) to the Eady model (Mukogawa and Ikeda 1994; Morgan 2001; Badger and Hoskins 2001) to low-resolution general circulation models (Buizza et al. 1993) to higher-resolution models (Buizza and Palmer 1995; Barkmeijer et al. 1998, 1999; Hoskins et al. 2000; Reynolds et al. 2001), the optimally growing structure typically is found in the midtroposphere, is subsynoptic in scale, and is dramatically tilted upshear. Though most share these features, the literature points out a few exceptions; for example, Buizza and Palmer (1995) and Reynolds et al. (2001) point out cases where the initial-time TE SV associated with the subtropical jet was maximized in amplitude near the tropopause.

Unfortunately, it seems possible that the initial-time TE SVs may not resemble the AEC SVs, and thus the TE SVs may have a relatively small expected projection onto the subspace defining the analysis-error covariances. For example, while TE SVs have largest amplitude in the midtroposphere, studies such as Hollingsworth and Lönnberg (1986) have shown that 6-h forecast errors (and, thus, presumably analysis errors) are largest near the tropopause and much smaller in the midtroposphere.

Because of such discrepancies, Barkmeijer et al. (1998, 1999) explored an alternative singular vector calculation, known as "Hessian SVs." The initial-time Hessian SVs are consistent with the analysis-error statistics implied by the observational network and the background-error covariances assumed in the three-dimensional variational assimilation scheme (3DVAR). These error statistics are stationary except to the extent that the observation network varies with time. In their studies, they compared the structure of the initial perturbations and the accuracy of subsequent probabilistic forecasts against those from TE SVs. The leading initialtime Hessian SVs were larger in scale than corresponding initial-time TE SVs, and their amplitude both at the initial time and optimization time was largest near the tropopause. Hessian SVs typically grew somewhat slower than TE SVs. The Hessian SVs had smaller initial amplitudes over data-rich continents and larger amplitudes over the oceans, consistent with the analysis-error statistics. Though the Hessian SVs had some desirable characteristics, probabilistic forecasts from TE SVs were found to be slightly more skillful. The exact reasons for this were unclear; Barkmeijer et al. (1999) hypothesized that perhaps the broad and deep correlation functions in the background-error covariance model used in 3DVAR unrealistically de-emphasized synoptic and subsynoptic scales, and that with a more realistic error covariance, the Hessian SV structures might more closely resemble those from TE SVs. Gelaro et al. 
(2002) recently performed a study of singular vectors using an analysis-error variance (not covariance) norm and found structures that were somewhat more similar to TE SVs than in the Barkmeijer et al. studies.

To date, no study has documented the structure of flow-dependent AEC SVs. The emergence of ensemblebased data assimilation methods (see, e.g., Evensen 1994; Houtekamer and Mitchell 1998, 2001; Burgers et al. 1998; Mitchell and Houtekamer 2000; Hamill et al. 2001; Whitaker and Hamill 2002; Mitchell et al. 2002) provides a vehicle for quantitatively exploring their approximate characteristics. These methods are based on an ensemble of parallel data assimilation cycles. Background-error covariances used during the data assimilation are modeled from the collection of ensemble forecasts. If care is taken to avoid filter divergence (see Hamill et al. 2001, and references therein), this ensemble of initial conditions should sample the distribution of analysis errors. Using this ensemble, one can readily approximate a few leading AEC SVs. This calculation involves finding the forecast structures that account for the most error variance in the space spanned by the ensemble. Under the assumption of linear error evolution, the initial-time AEC SV is computed by applying to the initial ensemble the same linear combination of ensemble members that produced the forecast structure with the largest variance. Attractively, this computation can be done without use of the linear tangent and adjoint versions of the forecast model.

A similar technique can be used to generate the approximate TE SVs. Following Hamill et al. (2000), we will compute approximate TE SVs using a very large (1600 member) ensemble of initial conditions composed of the ensemble mean analysis plus random perturbations consistent with an initial total-energy norm. A singular value decomposition of the resulting forecasts then determines the forecast structures with the most variance as well as the initial perturbations that will evolve into those forecast structures.

This paper will focus on a description of the structure of these AEC SVs and a comparison with TE SVs. Though it is an important question for ensemble forecasting, we will not address in this paper the question of the relative usefulness of random and singular vector ensembles; we hope to address this in our future work.

The paper is organized as follows. Section 2 provides a brief description of the forecast model and the ensemble data assimilation methodology. Section 3 describes the methodology for generating flow-dependent AEC SVs and the approximate TE SVs. The details of our experiment and test methodology are described in section 4. Section 5 describes the characteristics of analysis errors, while section 6 provides results, and section 7 a discussion and conclusions.

\section{Ensemble-based data assimilation methodology}

\section{a. Forecast model}

A T31 L15 dry primitive equation spectral model will be used in the following experiments. There are no ter- rain features nor surface variations (however, in subsequent plots we will superimpose maps of the earth's coastlines for reference). The model has 47150 degrees of freedom. The prognostic variables are vorticity, divergence, temperature, and surface pressure. Except for a minor modification to the forcing, described below, the model is essentially equivalent to the model of Held and Suarez (1994).

The experiment is conducted under perfect-model assumptions; that is, the same forecast model is used both to generate a synthetic true state and the ensemble forecasts. To generate a time series of the true state, we started with a random perturbation superimposed upon a resting state. The model was then integrated for 280 days. The first 100 days were discarded, and the remaining 180 days taken as the time series of the true state used in this experiment. Hereafter, day 100 is considered the starting point, the day 0 for all further experiments.

As described in Hamill et al. (2002b), our initial experiments with this model using the classic Held and Suarez (1994) forcing frequently produced low-quality analyses in the tropical upper troposphere. After much examination, we concluded that the root cause was small-scale wave motions excited under adiabatic or superadiabatic conditions. We tested the use of linear vertical diffusion to control this noise, but this was largely ineffective. As noted by Bénard et al. (2000), nonlinear vertical diffusion schemes are preferable but must be coded very carefully to ensure stability. We decided as an alternative to modify the radiative equilibrium state to which temperature is relaxed, making it slightly more stable in the tropical upper troposphere. The classic Held and Suarez equilibrium state is defined by

$$
\begin{aligned}
T_{\text {eq }}=\max \{200 K, & {\left[315 K-(\Delta T)_{y} \sin ^{2} \phi\right.} \\
& \left.\left.-(\Delta \theta)_{z} \log \left(\frac{p}{p_{0}}\right) \cos ^{2} \phi\right]\left(\frac{p}{p_{0}}\right)^{\kappa}\right\},
\end{aligned}
$$

where $(\Delta T)_{y}=60 \mathrm{~K}, \phi$ is the latitude, $(\Delta \theta)_{z}=10 \mathrm{~K}$, $p_{0}=1000 \mathrm{hPa}, \kappa=R / c_{p}=2 / 7$, and $c_{p}=1004 \mathrm{~J} \mathrm{~kg}^{-1}$ $\mathrm{K}^{-1}$. Our modified Held-Suarez forcing is of the form

$$
\begin{aligned}
T_{\mathrm{eq}}=\max \{200 K, & {\left[315 K-(\Delta T)_{y} \sin ^{2} \phi\right.} \\
& \left.\left.-(\Delta \theta)_{z} \log \left(\frac{p}{p_{0}}\right)\left(1+\cos ^{2} \phi\right)\right]\left(\frac{p}{p_{0}}\right)^{\kappa}\right\} .
\end{aligned}
$$

The change in the equilibrium state between the two is illustrated in Fig. 1.

\section{b. Ensemble data assimilation methodology}

The assimilation scheme used here has been named the ensemble square root filter (EnSRF). A complete 

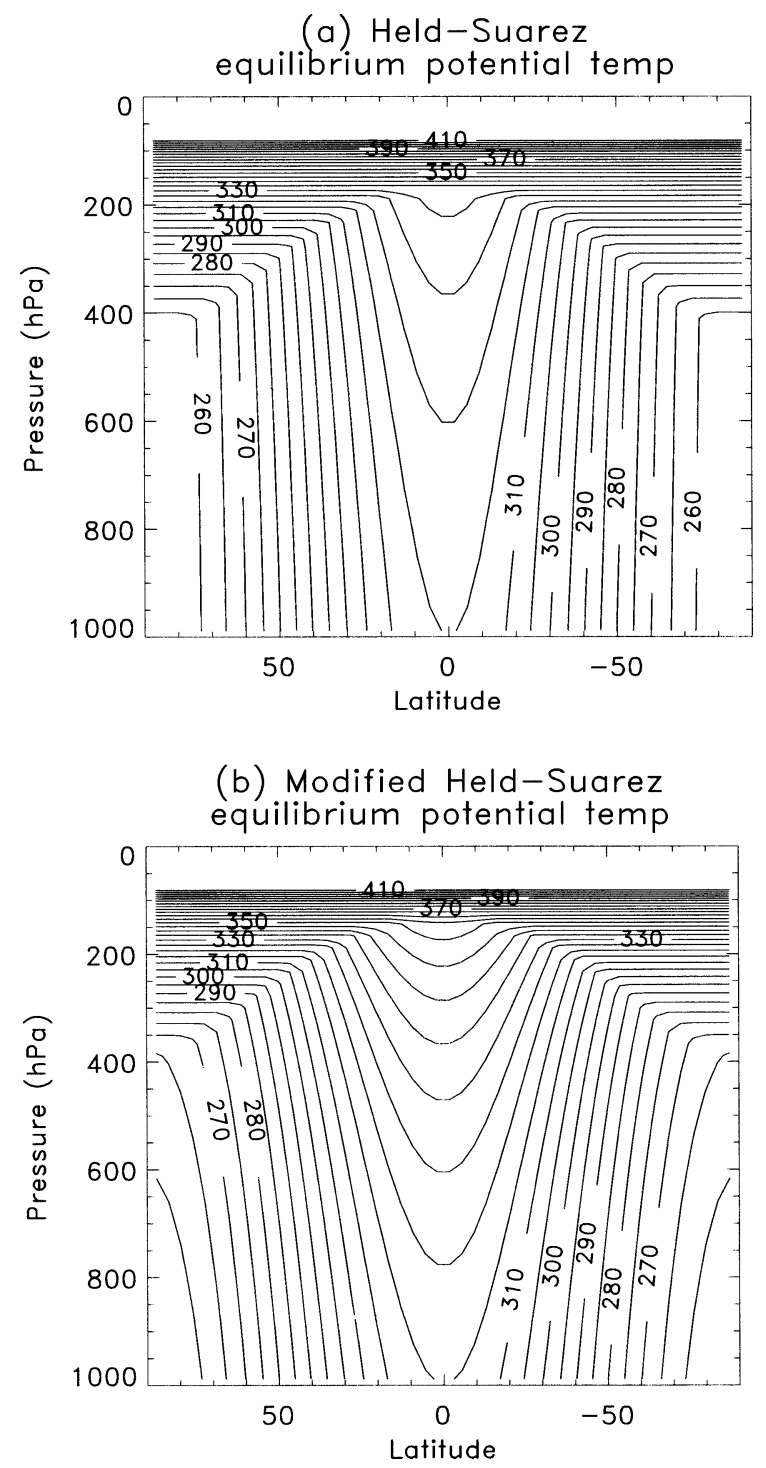

FIG. 1. (a) Classic Held-Suarez potential temperature profile; (b) modified Held-Suarez potential temperature profile (with more stable tropical troposphere) used here. Temperature in K. Contours are not plotted above $410 \mathrm{~K}$.

description of it and the rationale for its use is provided in Whitaker and Hamill (2002). The underlying principle is to run an ensemble of parallel forecast and data assimilation cycles, ensuring that the ensemble mean analysis and the analysis-error covariance as estimated by the ensemble are consistent with that predicted by Kalman filter theory.

We follow the notational convention of Ide et al. (1997). Let $\mathbf{x}^{b}$ be a background model forecast, $\mathbf{y}^{o}$ be a set of observations, and $\mathbf{H}$ be an operator that converts the model state to the observation space, here assumed linear. Let $\mathbf{P}^{b}$ be the background-error covariance matrix and $\mathbf{R}$ be the observational-error covariance matrix. The minimum error variance estimate of the analyzed state $\mathbf{x}^{a}$ is then given by the traditional Kalman filter update equation (Lorenc 1986)

$$
\mathbf{x}^{a}=\mathbf{x}^{b}+\mathbf{K}\left(\mathbf{y}^{o}-\mathbf{H} \mathbf{x}^{b}\right),
$$

where

$$
\mathbf{K}=\mathbf{P}^{b} \mathbf{H}^{\mathrm{T}}\left(\mathbf{H} \mathbf{P}^{b} \mathbf{H}^{\mathrm{T}}+\mathbf{R}\right)^{-1} .
$$

The expected analysis-error covariance is

$$
\mathbf{P}^{a}=(\mathbf{I}-\mathbf{K H}) \mathbf{P}^{b} .
$$

In the standard ensemble Kalman filter (EnKF; Evensen 1994; Houtekamer and Mitchell 1998; Burgers et al. 1998; Hamill et al. 2001), parallel data assimilation cycles are conducted, each cycle updating a member background forecast to a set of perturbed observations perturbed with noise consistent with observational errors. Here, $\mathbf{P}^{b}$ is approximated using the sample covariance from an ensemble of model forecasts; $\mathbf{P}^{b}=\left\langle\mathbf{x}^{\prime b} \mathbf{x}^{\prime b \mathrm{~T}}\right\rangle$, where primes denote the deviation from the ensemble mean and $\langle\cdot\rangle$ denotes the expected value, here computed from the ensemble. In actuality, there is no need to compute and store the full matrix $\mathbf{P}^{b}$. Instead, matrices $\mathbf{P}^{b} \mathbf{H}^{\mathrm{T}}$ and $\mathbf{H P}^{b} \mathbf{H}^{\mathrm{T}}$ are estimated directly using the ensemble (Evensen 1994; Houtekamer and Mitchell 1998).

The EnSRF operates similarly, conducting a set of parallel data assimilation cycles. It is convenient in the EnSRF to update the equations for the ensemble mean (denoted by an overbar) and the deviation from the mean separately:

$$
\begin{aligned}
\overline{\mathbf{x}}^{a} & =\mathbf{x}^{b}+\mathbf{K}\left(\mathbf{y}^{o}-\mathbf{H} \overline{\mathbf{x}}^{b}\right), \\
\mathbf{x}_{i}^{\prime a} & =(\mathbf{I}-\tilde{\mathbf{K}} \mathbf{H}) \mathbf{x}_{i}^{\prime b} .
\end{aligned}
$$

Here, $\mathbf{K}$ is the traditional Kalman gain given by Eq. (4), and $\mathbf{K}$ is the "reduced" gain used to update deviations from the ensemble mean.

When sequentially processing independent observations, $\mathbf{K}, \tilde{\mathbf{K}}, \mathbf{H} \mathbf{P}^{b}$ and $\mathbf{P}^{b} \mathbf{H}^{\mathrm{T}}$ are all vectors with the same number of elements as the model state vector, and $\mathbf{H P}^{b} \mathbf{H}^{\mathrm{T}}$ and $\mathbf{R}$ are scalars. Thus, when observations are processed one at a time,

$$
\tilde{\mathbf{K}}=\left(1+\sqrt{\frac{\mathbf{R}}{\mathbf{H P}^{b} \mathbf{H}^{\mathrm{T}}+\mathbf{R}}}\right)^{-1} \mathbf{K} .
$$

The quantity multiplying $\mathbf{K}$ in Eq. (8) is thus a scalar between 0 and 1 . This means that, in order to obtain the desired analysis-error covariance, one updates deviations from the ensemble mean using a modified Kalman gain that is reduced in magnitude relative to the traditional Kalman gain. Thus, deviations from the mean are reduced less in the analysis using $\tilde{\mathbf{K}}$ than they would be using $\mathbf{K}$. In the EnKF, the excess variance reduction caused by using $\mathbf{K}$ to update deviations from the mean is compensated for by the introduction of noise to the observations. In the EnSRF, the mean and departures from the mean are updated independently according to Eqs. (6) and (7). If observations are processed one at a 
time, the EnSRF requires about the same computation as the traditional EnKF with perturbed observations.

The general analysis methodology is thus as follows. Generate a set of perturbed initial conditions. Make $n$ forecasts forward to the next data assimilation time. Perform $n+1$ parallel data assimilation cycles, updating the mean state using Eqs. (6) and (4) and the $n$ perturbations using Eqs. (7) and (8). Repeat the process. In each data assimilation cycle, observations are assimilated serially.

Some additional algorithmic complexity will be used in order to model background-error covariances more accurately. These include the inflation and localization of covariances. Deviations of perturbations of each member from the ensemble mean are inflated by a small amount before the start of each data assimilation cycle in order to ensure that covariances are not systematically underestimated. Such underestimation can cause a problem known as filter divergence, whereby the influence of new observations is ignored. Covariance localization multiplies the ensemble estimate of covariances with an isotropic function that monotonically decreases with greater distance from the observation. See Hamill et al. (2001) and references therein for an in-depth rationale and mathematical formalism.

\section{Approximate singular vectors generated from ensembles}

\section{a. Analysis-error covariance singular vectors}

Before describing how the leading AEC SVs can be approximated given an ensemble of analyses from the EnSRF, we first briefly review the definition and some properties of the AEC SVs. We use the following notational conventions. Here, $\mathbf{S}$ denotes a symmetric, nonnegative matrix defining a norm for the forecast errors by $|\mathbf{x}|^{2}=\mathbf{x}^{\mathrm{T}} \mathbf{S x}$. In what follows, we will define $|\cdot|$ to be the total energy norm and write $\mathbf{S}=\mathbf{D}^{\mathrm{T}} \mathbf{D}$, where D is a matrix that transforms and scales the state vector so that $(\mathbf{D x})^{\mathrm{T}} \mathbf{D} \mathbf{x}$ is the total energy of $\mathbf{x}$. We will use $\mathbf{M}$ $=\mathbf{M}\left(t_{1}, t_{2}\right)$ to denote the resolvent of the tangent linear dynamics over the interval $\left[t_{1}, t_{2}\right]$ (i.e., $\mathbf{M}$ maps sufficiently small analysis error at $t=t_{1}$ onto forecast errors at $t=t_{2}$ ).

To find the analysis-error covariance singular vectors, we would like to maximize the forecast perturbation $\mathbf{x}^{\prime f}$, measured in a total-energy norm, subject to the constraint that the initial perturbations $\mathbf{x}^{\prime a}$ are sized to be consistent with the analysis-error statistics, that is,

$$
\max \frac{\mathbf{x}^{\prime f^{\mathrm{T}}} \mathbf{S} \mathbf{x}^{\prime f}}{\mathbf{x}^{\prime a^{\mathrm{T}}} \mathbf{P}^{a-1} \mathbf{x}^{\prime a}} \quad \text { or } \quad \max \frac{\mathbf{x}^{\prime a^{\mathrm{T}}} \mathbf{M}^{\mathrm{T}} \mathbf{S} \mathbf{M} \mathbf{x}^{\prime a}}{\mathbf{x}^{\prime a^{\mathrm{T}}} \mathbf{P}^{a-1} \mathbf{x}^{\prime a}}
$$

The normalization in the denominator ensures that the size and structure of $\mathbf{x}^{\prime a}$ are consistent with those of a perturbation drawn from a normal distribution with zero mean and covariance $\mathbf{P}^{a}$. Thus, when the denominator is large, the error is unlikely. By Rayleigh's principle, these AEC SVs are also a solution to the generalized eigenvalue problem

$$
\mathbf{M}^{\mathrm{T}} \mathbf{S M u}=\lambda \mathbf{P}^{a-1} \mathbf{u},
$$

where $\mathbf{u}$ denotes an eigenvector and $\lambda$ the associated eigenvalue (also see Barkmeijer et al. 1998).

The significance of the solutions $\mathbf{u}$ in Eq. (9) can be seen by defining $\mathbf{v}=$ DMu. Multiplying Eq. (9) on the left by $\mathbf{D M P} \mathbf{P}^{a}$ and recalling that $\mathbf{S}=\mathbf{D D}^{\mathrm{T}}$ then yields

$$
\mathbf{D M P} a \mathbf{M}^{\mathrm{T}} \mathbf{D}^{\mathrm{T}} \mathbf{v}=\lambda \mathbf{v} \text {. }
$$

The forecast error covariance at the optimization time is $\mathbf{P}^{f}=\left\langle\mathbf{x}^{\prime f} \mathbf{X}^{\prime f^{\mathrm{T}}}\right\rangle$. If analysis errors evolve linearly, then $\mathbf{P}^{f}=\mathbf{M} \mathbf{P}^{a} \mathbf{M}^{\mathrm{T}}$ and Eq. (10) becomes

$$
\mathbf{D P}_{f} \mathbf{D}^{\mathrm{T}} \mathbf{v}=\lambda \mathbf{v} \text {. }
$$

Thus, the solutions $\mathbf{v}$ of Eq. (10) are the eigenvectors of the forecast-error covariance matrix (after transforming and scaling the state with D), and $\mathbf{u}$ in Eq. (9) is the initial error that evolves to produce $\mathbf{v}$, via $\mathbf{v}=\mathbf{D M u}$. We will refer to $\mathbf{u}$ as the initial-time AEC SV, since it corresponds to an error in the analysis, and to $\mathbf{v}$ as the evolved or forecast AEC SV, since it corresponds to the forecast error produced by the analysis error $\mathbf{u}$.

Now, we wish to approximate the leading AEC SVs using an ensemble of analyses at $t=t_{1}$ from the EnSRF and an ensemble of forecasts to $t=t_{2}$ from those analyses. Let $\mathbf{X}_{a}=(n-1)^{-1 / 2}\left[\mathbf{x}_{1}^{a}-\overline{\mathbf{x}}^{a}, \ldots, \mathbf{x}_{n}^{a}-\overline{\mathbf{x}}^{a}\right]$, where the $i$ th column vector represents the $i$ th member $\mathbf{x}_{i}^{a}$ 's analyzed model state deviation from the ensemble mean analysis $\overline{\mathbf{x}}^{a}$. Similarly, let $\mathbf{X}_{f}=(n-1)^{-1 / 2}\left[\mathbf{x}_{1}^{f}-\right.$ $\left.\overline{\mathbf{x}}^{f}, \ldots, \mathbf{x}_{n}^{f}-\overline{\mathbf{x}}^{f}\right]$, where $\mathbf{x}_{i}^{f}$ is the forecast from $\mathbf{x}_{i}^{a}$ based on the full (nonlinear) forecast model. In order to approximate the AEC SVs, the ensemble to be used must satisfy

$$
\lim _{n \rightarrow \infty} \mathbf{X}_{a} \mathbf{X}_{a}^{\mathrm{T}}=\mathbf{P}^{a} \quad \text { and } \quad \lim _{n \rightarrow \infty} \mathbf{X}_{f} \mathbf{X}_{f}^{\mathrm{T}}=\mathbf{P}^{f} ;
$$

that is, the sample covariance matrices based on the analysis and forecast ensembles must approximate $\mathbf{P}^{a}$ and $\mathbf{P}^{f}$, respectively, for sufficiently large ensembles.

The forecast AEC SVs can be approximated by the solutions of

$$
\mathbf{D X}_{f}(\mathbf{D X})_{f}^{\mathrm{T}} \mathbf{v}=\lambda \mathbf{v}
$$

This is because, given the second condition in Eq. (12), Eqs. (11) and (13) become identical for large $n$. We compute solutions of Eq. (13) by representing $\mathbf{v}$ as a linear combination of the $n$ forecast deviations, that is,

$$
\mathbf{v}=\mathbf{D} \mathbf{X}_{f} \mathbf{a} \text {. }
$$

Substituting for $\mathbf{v}$ in Eq. (13) and eliminating a factor of $\mathbf{D X}_{f}$ from each side, we obtain an equivalent (for $\lambda$ $\neq 0$ ) but smaller $n \times n$ eigenproblem,

$$
\left(\mathbf{D X}_{f}\right)^{\mathrm{T}} \mathbf{D} \mathbf{X}_{f} \mathbf{a}=\mathbf{X}_{f}^{\mathrm{T}} \mathbf{S} \mathbf{X}_{f} \mathbf{a}=\lambda \mathbf{a},
$$

whose solutions can be found by conventional numerical techniques. Alternatively, one may compute $\mathbf{v}$ as the left 
singular vectors in a singular value decomposition (SVD) of $\mathbf{D X} \mathbf{X}_{f}$. We have found negligible differences in the resulting numerical solutions.

To approximate the initial-time AEC SVs, we need a similar approximation to the solutions $\mathbf{u}$ of Eq. (9). This requires the assumption that the forecast-error evolution is approximately linear; in that case, $\mathbf{X}_{f}=\mathbf{M} \mathbf{X}_{a}$ and Eq. (15) becomes

$$
\mathbf{X}_{a}^{\mathrm{T}} \mathbf{M}^{\mathrm{T}} \mathbf{S} M \mathbf{X}_{a} \mathbf{a}=\lambda \mathbf{a} .
$$

Multiplying by $\mathbf{X}_{a}$ and setting $\mathbf{u}=\mathbf{X}_{a} \mathbf{a}$ then gives, for large $n$,

$$
\mathbf{P}^{a} \mathbf{M}^{\mathrm{T}} \mathbf{S} \mathbf{M X}{ }_{a} \mathbf{a}=\mathbf{P}^{a} \mathbf{M}^{\mathrm{T}} \mathbf{S M u}=\lambda \mathbf{u},
$$

which is identical to Eq. (9). Note that the leading eigenvectors a of Eq. (15) provide both an approximate initial AEC SV, through $\mathbf{u}=\mathbf{X}_{a} \mathbf{a}$, and an approximate evolved AEC SV, through $\mathbf{v}=\mathbf{D X}_{f} \mathbf{a}$, which is exactly Eq. (14).

Hence, our overall algorithm for computing the singular vectors from a large ensemble is as follows. Equation (15) is first solved to yield the appropriate linear combination a. The initial-time singular vectors are then readily computed as $\mathbf{u}=\mathbf{X}_{a} \mathbf{a}$, and the approximate evolved AEC SVs from $\mathbf{v}=\mathbf{D X}_{f} \mathbf{a}$. These singular vectors are approximations to the true singular vectors that can only be obtained if the error dynamics is truly linear and if the ensemble size is infinite. In section 6 we will provide some evidence that linear dynamics can appropriately be assumed and that the singular vector structure from ensembles larger than the $n=400$ used here should not change much.

\section{b. Total-energy singular vectors}

The technique for generating approximate TE SVs is very similar to that used to generate AEC SVs, differing only in the method of constructing the initial ensemble. For AEC SVs, the initial ensemble represented random samples of analysis error; for TE SVs, the initial ensemble consists of the mean analysis state plus very small random perturbations that are designed to be white in a total-energy norm. See also Hamill et al. (2000) for a similar computation in a quasigeostrophic model.

Let $\left(\mathbf{u}, \mathbf{v}, \mathbf{T}, \mathbf{P}_{s}\right)^{\mathrm{T}}$ denote a vector of wind, temperature, and surface pressure perturbations to the mean analysis state. We would like to generate perturbations that are white in total energy (have equal energy in all resolved scales) and that have a small fraction of the energy of the basic state so that perturbations will evolve linearly. Following Ehrendorfer and Errico (1995), the total energy of the perturbation is defined as

$$
\|\cdot\|=\frac{\sqrt{\frac{1}{2} \int_{E} \int_{0}^{1}\left[u^{2}+v^{2}+\frac{c_{p}}{T_{r}} T^{2}+R_{d} T_{r}\left(\frac{p_{s}}{p_{r}}\right)^{2}\right] d \sigma d E}}{\int_{E} \int_{0}^{1} d \sigma d E}
$$

where $E$ indicates the horizontal domain, $\sigma$ is the vertical coordinate, $T_{r}$ is a reference temperature (here, 300 $\mathrm{K}), R_{d}$ is the gas constant for dry air $\left(287 \mathrm{~J} \mathrm{~K}^{-1} \mathrm{~kg}^{-1}\right)$, $c_{p}$ is the specific heat of dry air at constant pressure (1004 $\left.\mathrm{J} \mathrm{K}^{-1} \mathrm{~kg}^{-1}\right), p_{s}$ is the surface pressure, and $p_{r}$ is a reference pressure $(1000 \mathrm{hPa})$.

Suppose we want to generate a random perturbation that is white in total energy. Our model is spectral, with a state vector consisting of vorticity, divergence, temperature, and surface pressure. Accordingly, let $V_{m n l}$, $D_{m n l}, T_{m n l}$, and $P_{m n}$ be perturbations to the vorticity, divergence, temperature, and pressure, respectively. Here, $m$ indicates the zonal wavenumber $(0-31), n$ the degree of the spherical harmonic, and $l$ the model level. Similar to Boer and Shepherd (1983), the total energy for our T31L15 model can be expressed as

$$
\begin{aligned}
\|\cdot\|=K_{\mathrm{te}} & \\
=\frac{1}{2} \sum_{m=-31}^{31} \sum_{n=|m|}^{31}\left[\sum_{l=1}^{15}(\right. & \alpha_{n} V_{m n l} V_{m n l}^{*}+\alpha_{n} D_{m n l} D_{m n l}^{*} \\
& \left.\left.+\beta T_{m n l} T_{m n l}^{*}\right)+\gamma P_{m n} P_{m n}^{*}\right],
\end{aligned}
$$

where $*$ denotes the complex conjugate, and coefficients $\alpha_{n}, \beta$, and $\gamma$ are defined as

$$
\alpha_{n}=\frac{r_{e}^{2}}{n(n+1)}, \quad \beta=\frac{c_{p}}{T_{r}}, \quad \text { and } \quad \gamma=\frac{R_{d} T_{r}}{p_{r}^{2}},
$$

where $r_{e}$ is the radius of the earth. To generate spectral perturbations that are small and white in total energy, we do as follows. 1) For each spectral component at each model level, generate independent random normal deviates for the real and imaginary components of $v_{m n l}$, $d_{m n l}, t_{m n l}$, and $p_{m n}$. 2) Scale the random normal deviates to generate perturbations to the state component.

Let

$$
\begin{aligned}
& V_{m n l}=k_{1} \alpha_{n}^{-1 / 2} v_{m n l}, \quad D_{m n l}=k_{1} k_{2} \alpha_{n}^{-1 / 2} d_{m n l}, \\
& T_{m n l}=k_{1} \beta^{-1 / 2} t_{m n l} \text {, and } P_{m n}=k_{1} \gamma^{-1 / 2} p_{m n} .
\end{aligned}
$$

Here, $k_{1}$ is a constant chosen so that the typical magnitude of the perturbation sums to $K_{t e}$, and $k_{2}$ is a ratio of the magnitude of divergence to vorticity perturbation size (here $k_{2}=0.2$; note that this decreases the divergent component of the wind, but because temperature and vorticity perturbations are generated independently, the initial perturbations are still largely unbalanced).

The overall methodology for calculating TE SVs at a particular time is as follows. First, calculate the ensemble mean of the EnSRF analyses. Next, generate an ensemble of perturbations that are white in total energy as described above. Add the perturbations to the mean analysis state, forming the ensemble of perturbed initial conditions. Integrate this ensemble forward $48 \mathrm{~h}$ using the fully nonlinear model. The methodology then follows for the AEC SVs: the linear combination of en- 


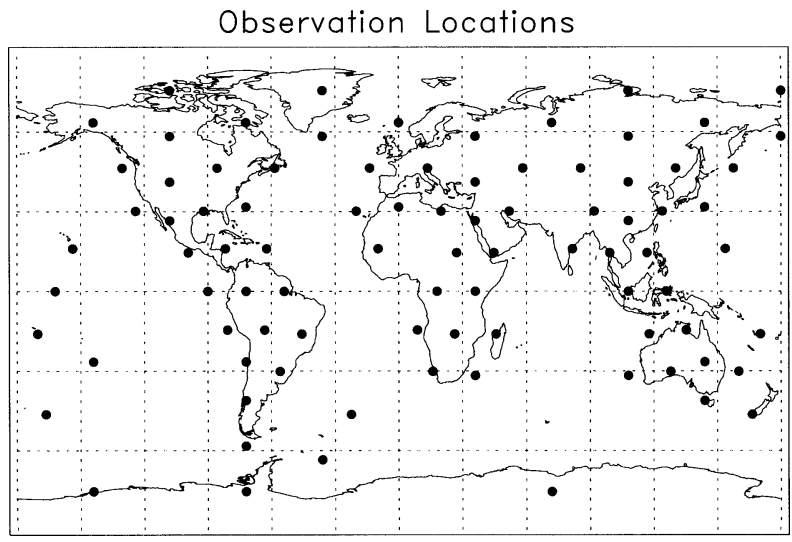

FIG. 2. Locations of synthetic rawinsonde profiles.

semble forecasts is found that results in the largest variance in total energy, and that same linear combination is applied to the initial ensemble to determine the leading initial-time TE SV.

Because the perturbations have a flat energy spectrum, a much larger ensemble of perturbations is needed to generate TE SV perturbations that grow rapidly. Even with a large ensemble, the initial structures are still much noisier than they are for the AEC SVs. See section 6 a for details.

\section{Experimental design}

Our experiment was conducted over an 180-day period and used a 400-member EnSRF data assimilation system as described in section $2 \mathrm{~b}$. In this implementation of the EnSRF, covariances were localized using a Schur product of ensemble covariances with an approximately Gaussian-shaped function with local support (Gaspari and Cohn 1999) reaching a zero value at 7000-km distance from the observation. Before each data assimilation cycle, covariances were inflated by $0.3 \%$.

Synthetic rawinsondes (raobs) were assimilated every $12 \mathrm{~h}$. The observations consisted of a surface pressure measurement and winds and temperatures at seven of the sigma levels, located approximately at 900, 766, 633, 500, 366, 233, and $100 \mathrm{hPa}$. Observations had error characteristics derived from Parrish and Derber (1992); wind errors were assumed to have a standard deviation of $1.73,2.18,2.7,2.8,3.2,3.0$, and $2.5 \mathrm{~m} \mathrm{~s}^{-1}$, respectively, at the seven levels, and temperature standard deviations of $1.6,1.4,1.3,1.3,1.9,2.5$, and $3.1 \mathrm{~K}$. Observation errors were assumed uncorrelated in the vertical. Observation locations are shown in Fig. 2; they were chosen to provide a crude analog to the operational raob network, with more observations over the "land" than the "ocean."

In order to examine the structure of AEC SVs, we periodically made an ensemble of 400 48-h forecasts from the 400 analyses. The first of these forecasts were conducted from the analyses on day 17.5, and another sample was generated every 5 days thereafter, yielding a total of 33 cases. The AEC SVs were computed using Northern Hemisphere ensemble forecast data north of $20^{\circ} \mathrm{N}$, and the set of evolved singular vectors was constructed to be orthogonal in the total-energy norm using the methodology outlined in section 3 . The evolved singular values and singular vectors were ordered from largest to smallest. To determine the associated initialtime structure, the linear combination of ensemble forecast members that produced a given evolved singular vector is used, but applied to the initial-time ensemble. Under linearity assumptions, this should produce the correct initial-time structure. The extent to which this assumption is valid will be examined in section 6 .

The method for generating approximate TE SVs was similar. A 1600-member ensemble of small initial perturbations that were white in energy was constructed and added to the mean analysis, followed by 160048 $\mathrm{h}$ forecasts.

\section{Analysis error characteristics}

Before examining the structure of the AEC SVs and TE SVs, we first document the characteristics of analysis errors, which are crucial in determining the AEC SV structure. A time series of the domain-average ensemble mean analysis error is shown in Fig. 3. Errors decreased quickly to a very low value, punctuated by occasional spikes of somewhat higher error. Figures $4 \mathrm{a}, \mathrm{b}$ show a zonal-mean cross section of the Northern Hemisphere ensemble mean analysis error. Wind errors were largest at the tropopause, with distinct maxima at three locations: near the equator, in the midlatitude jet core, and near the North Pole. The former was primarily due to small-scale wave motions generated when the atmosphere is adiabatic or superadiabatic; this maximum would have been substantially larger without the use of the modified Held-Suarez forcing discussed in section $2 \mathrm{a}$. The maximum in the midlatitudes was due to rapid error growth associated with the jet, and the maximum at the pole was due to a lack of observations. Temperature errors had a less complex structure, with a tropospheric maximum near the pole and a minimum in the tropical lower troposphere. Though we will focus on the Northern Hemisphere hereafter, where errors were very small, we note that the errors were generally much larger in the Southern Hemisphere, where the observational network was more sparse (Fig. 5).

A desirable property of an ensemble data assimilation system is that one should be able to consider the analyses and the true state as random samples from the same probability distribution. This can be evaluated with rank histograms (Hamill 2001, and references therein). Figure 6 shows selected rank histograms for several analysis variables. With the exception of temperatures in the tropical upper troposphere, the predominant characteristic of the analyses was a slight excess of spread in the ensembles, 


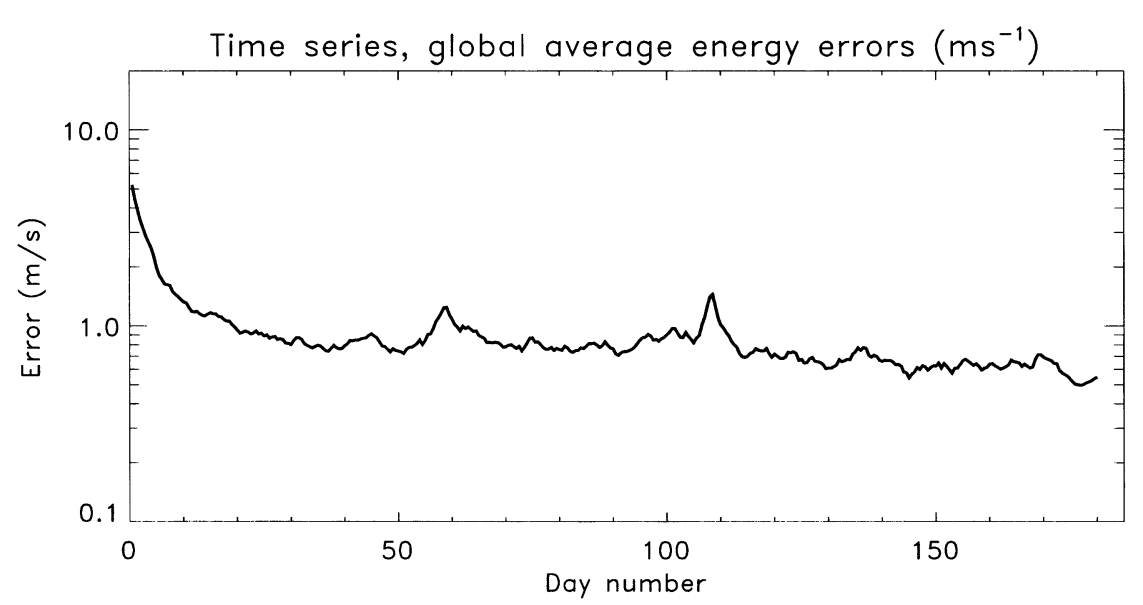

FIG. 3. Time series of errors of ensemble mean analysis from ensemble square root filter. Errors are expressed in a total-energy norm integrated over the domain.
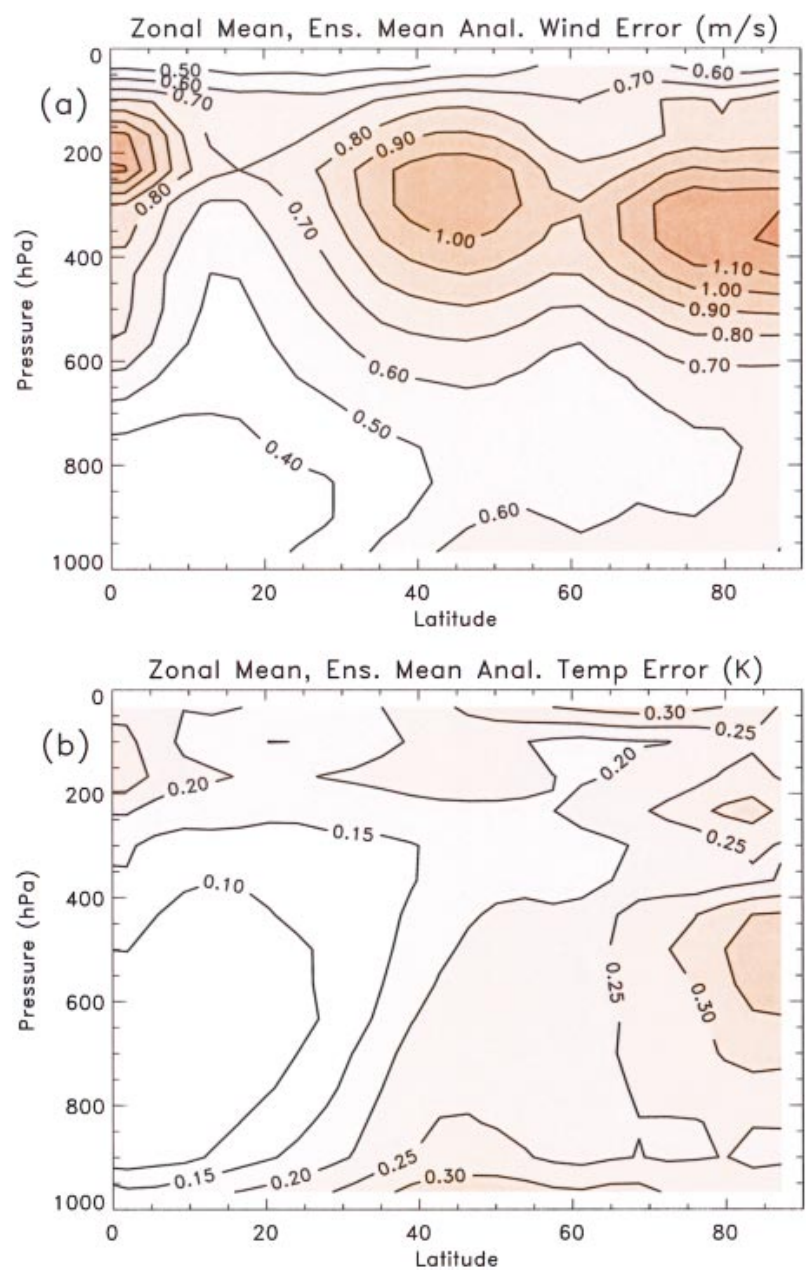

FIG. 4. Time- and zonally averaged ensemble mean absolute analysis error taken from samples between days 35 and 180: (a) wind error and (b) temperature error. manifested in a convex shape to the histograms. The apparent bias of tropical upper-tropospheric temperatures was initially a major concern, suggesting a problem such as filter divergence. However, as shown in Fig. 4, the ensemble mean temperature analysis error in the Tropics was exceedingly small. A map of zonally and temporally averaged temperature bias is presented in Fig. 7. The bias was also uniformly small; at $300 \mathrm{hPa}$ in the Tropics, the bias was $\sim 0.10 \mathrm{~K}$. Since the analysis error of temperatures was typically about $0.2 \mathrm{~K}$ or less in the Tropics (Fig. 4), this tiny bias had a large effect on the rank histograms. Since our concern will be primarily in the Northern Hemisphere midlatitudes where rank histograms were generally more uniform, we conclude that the ensemble should generally be useful for providing random samples of analysis error.

These results are broadly consistent with those of results of Mitchell et al. (2002), who conducted experiments in a similar model with a similar data assimilation scheme.

\section{Results}

\section{a. Characteristics of singular vectors}

We first consider the growth rate of the ordered AEC SVs, shown in Fig. 8a. Averaged over many cases, the energy of the leading singular vector grew by a factor of slightly less that 4 during the 48 -h forecast. Since energy is a squared quantity, this implies that perturbations approximately doubled in size during the $48 \mathrm{~h}$. Though this growth rate may seem small, the error doubling time for this model at this resolution is around 4 days (higher-resolution versions of this model have faster, more realistic error doubling times). Thus, the leading singular vector grew approximately twice as fast as the leading Lyapunov vector (Legras and Vautard 1996).

In this analysis, the singular vectors were ordered by the amount of forecast error variance that they explain. 


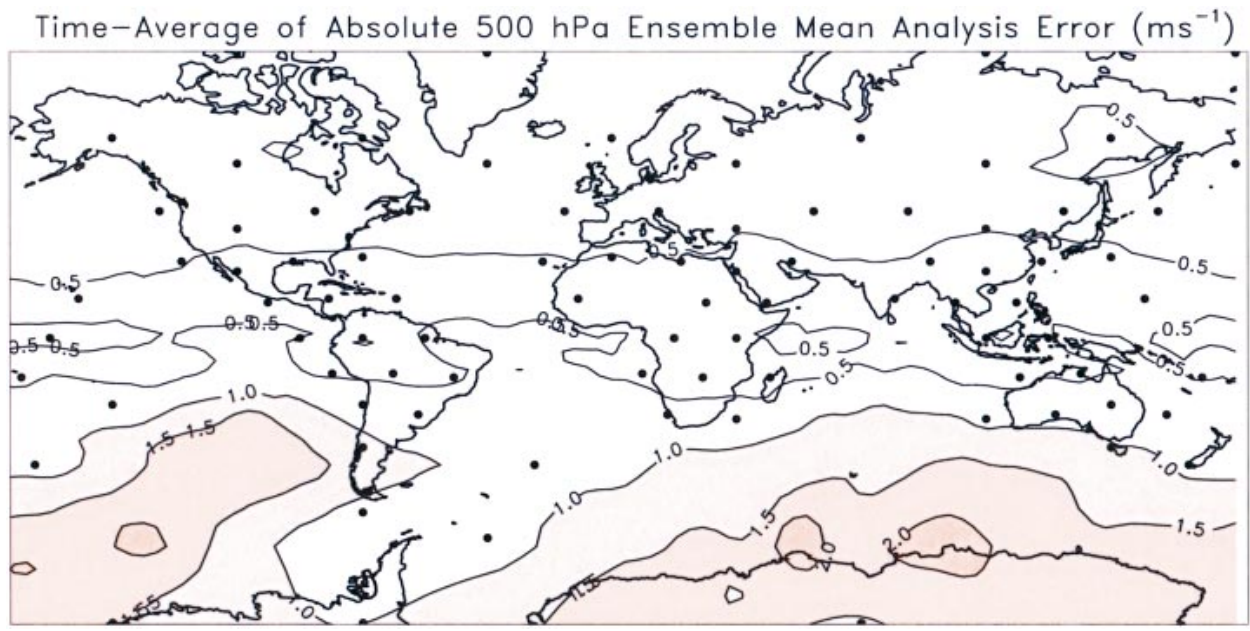

FIG. 5. Map of time-averaged ensemble mean error in energy norm at $500 \mathrm{hPa}$. Locations of raobs are overplotted.

This does not imply that the leading AEC SV was necessarily associated with the fastest growing structure. The largest forecast error could have been due to rapid error growth, but it may also have been due to the initial analysis structure having relatively large errors in that direction in phase space. Such a contingency is illustrated by Fig. 8b, showing the growth rates for an individual case (day 132.5). Here, singular vector 3 grew faster than both singular vectors 1 and 2 .

The TE SVs grew faster on average than AEC SVs, and there were more growing directions (Fig. 8c). This is broadly consistent with the results of Barkmeijer et al. (1998, 1999), who found that their TE SVs grew much faster than the Hessian SVs. Note that it is quite likely that with a larger ensemble, yet faster growth of the TE SVs would be possible. When the amplification of the leading singular vector was calculated from a 100 -member ensemble, this was approximately 1.7 ; for a 400-member ensemble, approximately 4.3; and as shown for the 1600-member ensemble, approximately 8.5. Still, the 1600 members were enough to verify that the features of these TE SVs were consistent with those observed in prior studies, as will be documented below.

Consider the average vertical structure of the leading singular vector. Figures 9a,b illustrate the zonal-mean structure of the leading AEC SV for the initial time and evolved (48 h). Amplitudes at both times were maximized in midlatitudes near the tropopause, with a secondary maximum near the poles, where the analysis errors were consistently large due to lack of data. There was also a secondary maximum in midlatitudes near the surface. The structure was qualitatively unlike that of TE SVs, shown in Figs. 9c,d. Consistent with previous studies (e.g., Buizza and Palmer 1995), the TE SVs started with a maximum initial amplitude in the midtroposphere. The initial structures of these TE SVs were
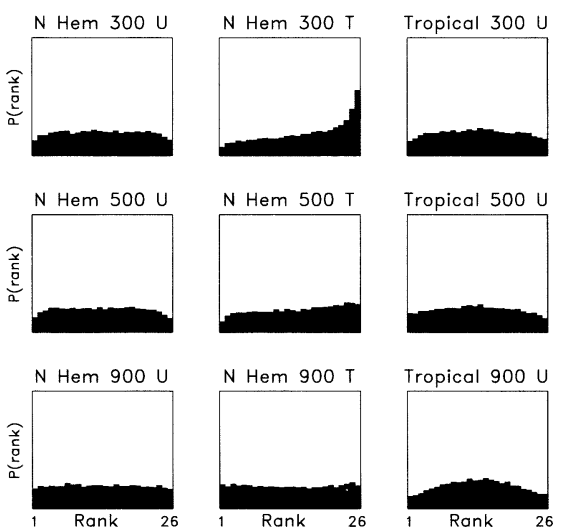
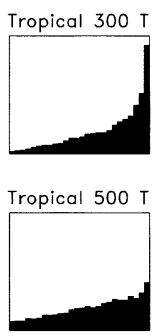

Tropical 900

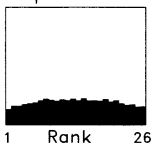

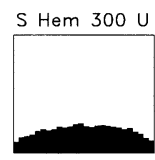

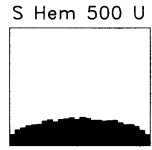

S Hem $900 \mathrm{U}$

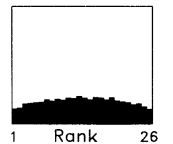

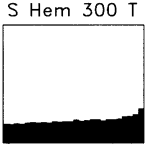

S Hem 500

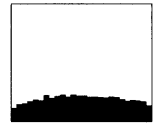

S Hem $900 \mathrm{~T}$

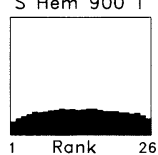

FIG. 6. Rank histograms of analysis errors. Rank histograms were generated using a subsample of 25 of the 400 ensemble members. Northern Hemisphere rank histograms used samples at every grid point between $30^{\circ}$ and $65^{\circ} \mathrm{N}$ latitude and every $15^{\circ}$ longitude. Tropical rank histograms used samples between $15^{\circ} \mathrm{N}$ and $15^{\circ} \mathrm{S}$ latitude, and Southern Hemisphere samples between $30^{\circ}$ and $65^{\circ} \mathrm{S}$ latitude. 


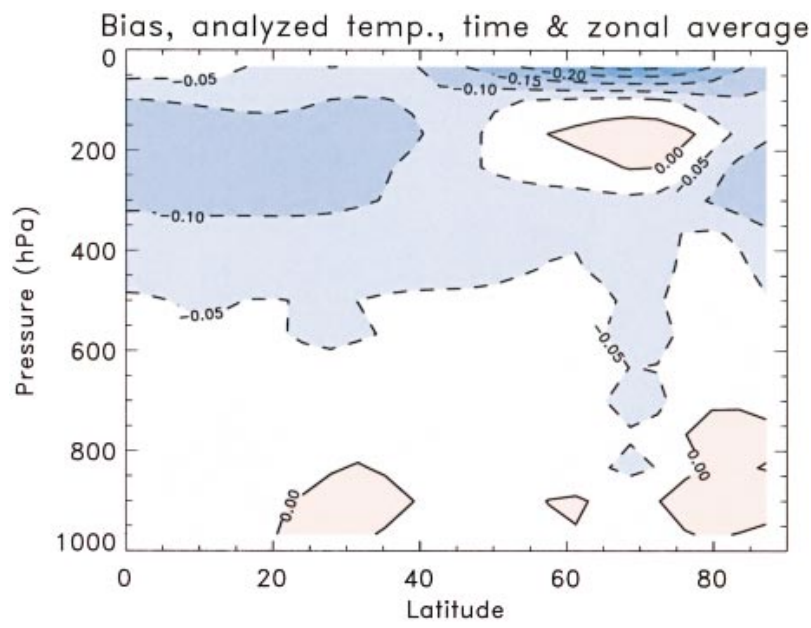

FIG. 7. Zonal average of bias of the ensemble mean analysis. Average was taken from days 35 to 180 .

not as well defined in this model as they were for experiments where the singular vectors were calculated using the linear tangent and adjoint; the leading singular vector contains both the relevant growing dynamical structure (e.g., Figs. 10,11) and spurious noise (hence the red color throughout the plot in Fig. 9c).

The growth of various components of the AEC SVs was also examined. In terms of individual wind components (not shown), the AEC SVs at both initial and evolved times were dominated by the meridional wind component, with much less amplitude in the zonal wind and temperature components. Temperature perturbations were found to grow primarily near the lower boundary, with less growth aloft. The initial-time TE SV temperature perturbations were much larger than the wind perturbations, were tilted upshear, and were maximized in the lower to midtroposphere (Fig. 10a), consistent with prior TE SV studies. Evolved TE SVs had larger-amplitude perturbations in wind than in temperature, and were deeper (Fig. 10b) and larger in scale (Fig. 11), again consistent with prior studies such as Barkmeijer et al. (1999).

The typical horizontal structure was also unlike the structure of TE SVs. The power spectrum of the leading singular vector (Fig. 12) shows that power was peaked around wavenumber 8 for both the initial-time and the evolved AEC SVs. The growth rate of the perturbations was roughly similar at all scales up to wavenumber 25 . This, again, was dramatically different than the TE SVs (Fig. 12 again), which initially have a larger fraction of their power at smaller scales but evolve to have a majority of the power at larger scales. This is consistent again with Buizza and Palmer (1995) and Barkmeijer et al. (1999, their Fig. 1).

Figures 13a,b show one case day's AEC SV structure at $300 \mathrm{hPa}$ at the initial and evolved times. The AEC SVs were synoptic in scale and had significant projections at most longitudes. The TE SVs in our model (e.g., Fig. 11) and those documented in prior studies were
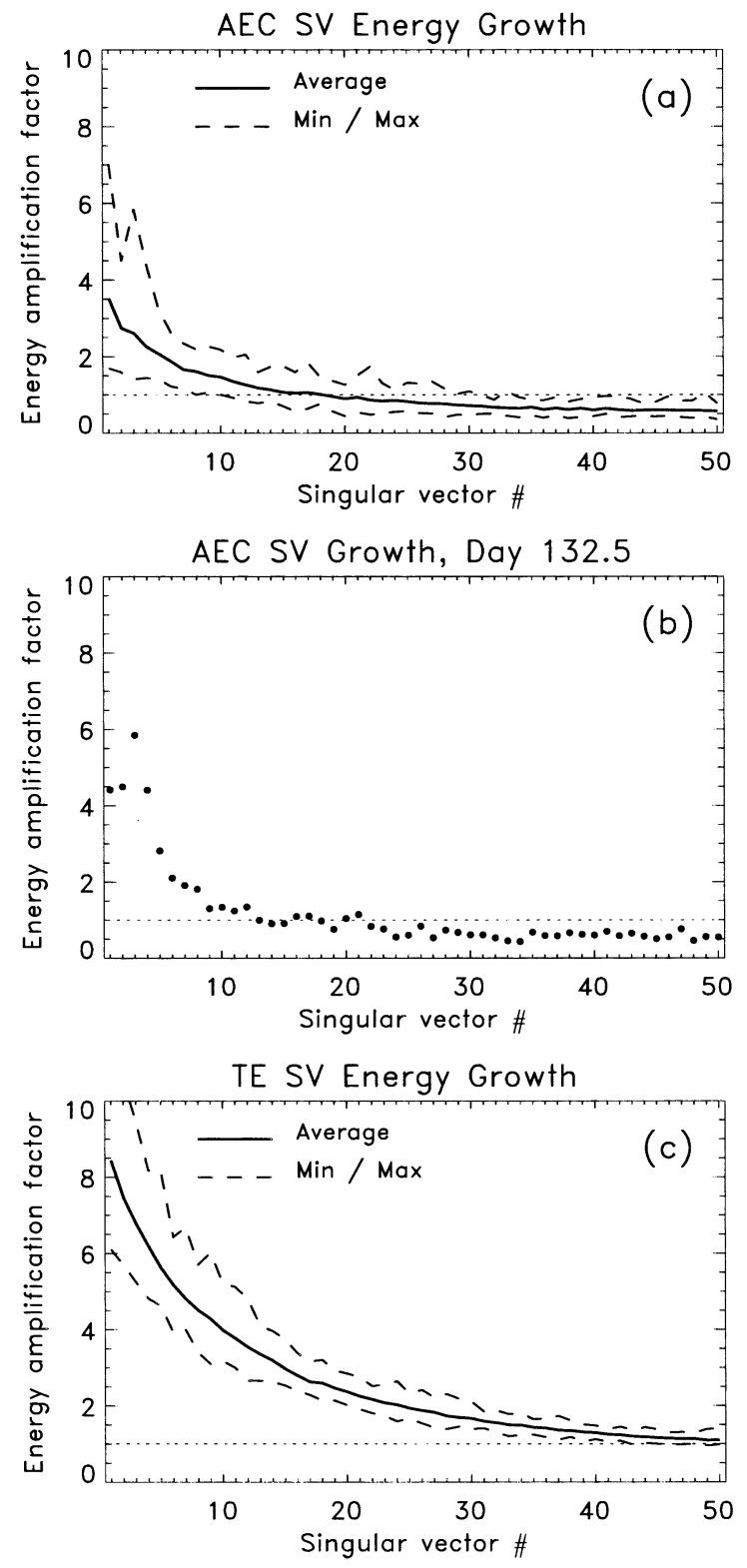

FIG. 8. (a) Growth rate (in total energy) over 48-h optimization period of AEC SVs as a function of singular vector number. Solid line denotes average over 33 cases, dashed lines the min and max from the 33 cases. (b) Example of an individual case where growth rates of AEC SV do not monotonically decrease with increasing singular vector number. (c) As in (a), but for TE SVs.

much more localized. Our first thought was that the nonlocality of the AEC SVs might have been a result of the insufficient ensemble size; perhaps 400 members was not enough to converge to a correct, more localized structure. However, visual comparisons of the leading AEC SV from 25- and 100-member ensembles suggest that the global nature of the singular vector does not appear to decrease with increasing sample size (Fig. 14). Perhaps the global structure could be an artifact of the simplicity of the forecast model, which does not have 

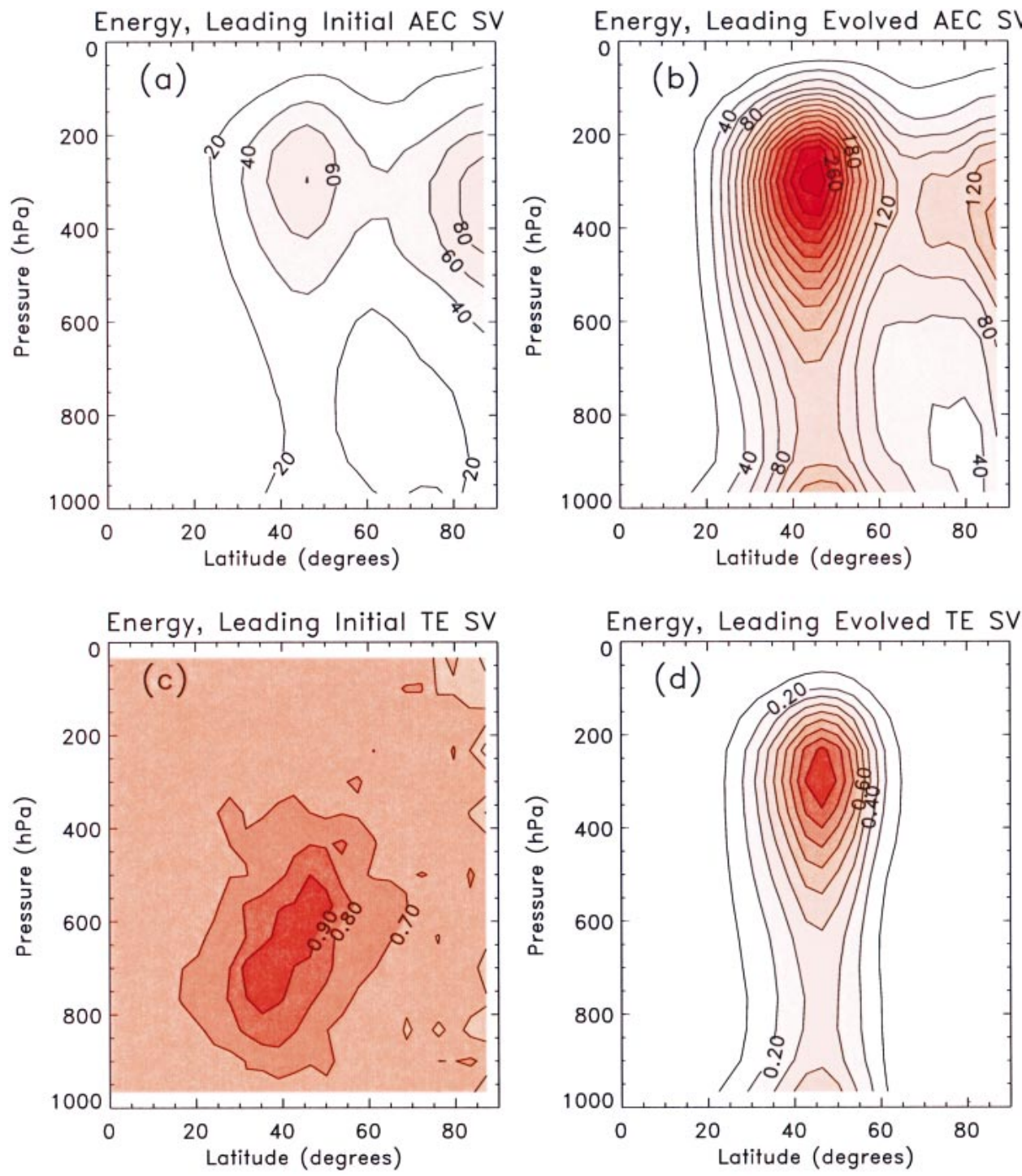

FIG. 9. Zonal-mean profile of energy of the leading AEC SV averaged over the 33 cases: (a) initial time and (b) 48-h evolved; units are $\mathrm{m}^{2} \mathrm{~s}^{-2}$ (c), (d) As in (a), (b) but for 48-h evolved TE SV; amplitudes are nondimensional energy; (c) chosen to be normalized by the maximum initial-time amplitude and (d) by the maximum final-time amplitude.

land-water interfaces or terrain features to regionalize areas of preferred cyclogenesis.

The evidence provided here suggests that the structure of flow-dependent AEC SVs is markedly different than that of TE SVs. Does this make AEC SVs a better basis for ensemble forecasting applications? Perhaps so, at least for shorter-range ensemble forecasts. Figure 15 provides a comparison of the projection of the error of the 48-h ensemble mean forecast onto the subspace of the leading 48-h forecast TE and AEC SVs for each of the 33 cases. As shown, the projection into the subspace of the AEC SVs is typically larger than into the subspace of TE SVs, suggesting that they are a more appropriate basis. A suggestion of why this may be so can be seen from a re-examination of Figs. 9b,d. The structure of the leading forecast AEC SV has significant amplitude near the poles, while this is missing in the leading TE SV. Recall that a large forecast error can result from either rapid growth of initially small errors or the persistence of large errors from the analysis. Error structures caused by this latter effect are naturally captured by AEC SVs and missed by TE SVs. Presumably, the more spatially homogeneous and spectrally white the initial errors, the greater the similarity of forecast AEC SVs and TE SV structures.

\section{b. Sampling and linearity issues}

We have not yet examined some of the underlying assumptions for creating these AEC SVs. There were 

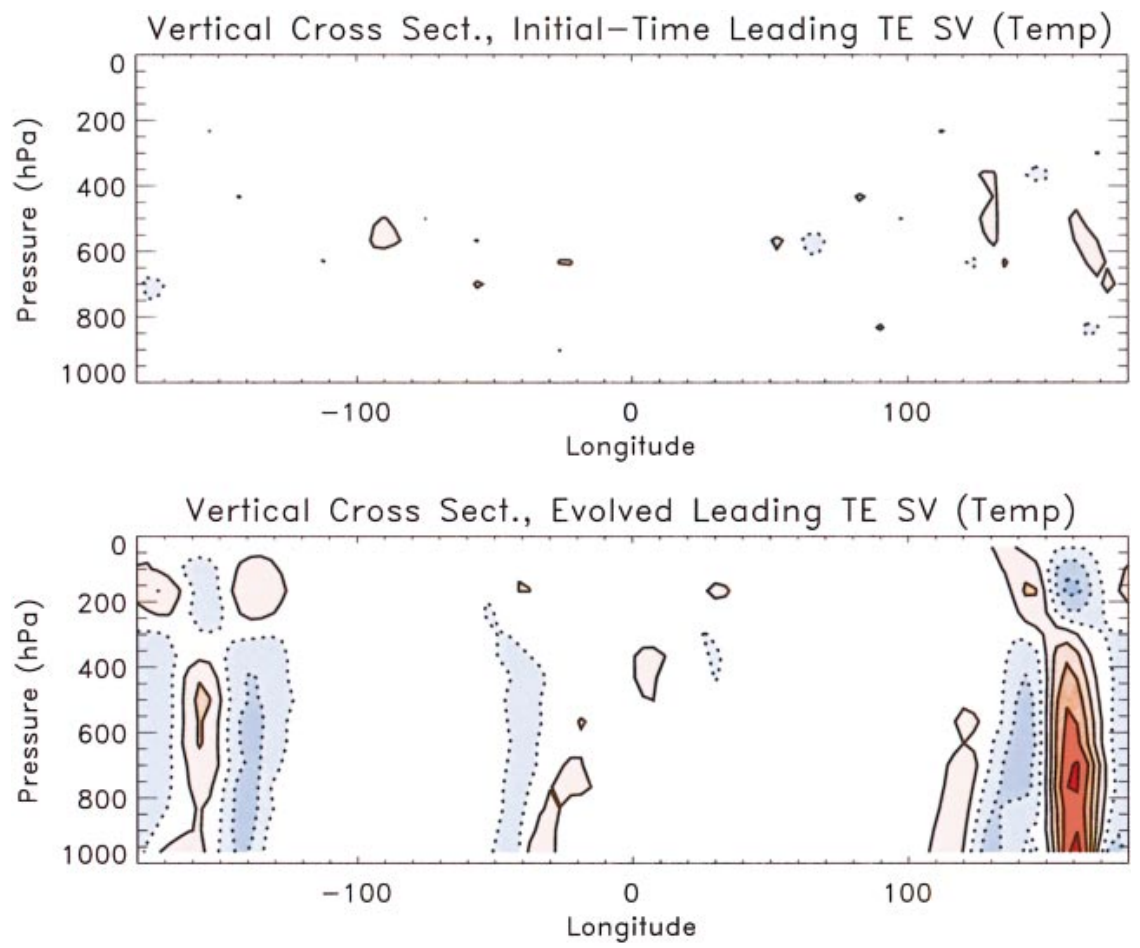

FIG. 10. Cross section of the temperature perturbation structure of the leading TE SV initialized on day 32.5. Cross section is along $\sim 42.5^{\circ} \mathrm{N}$ latitude. Perturbations are normalized by the magnitude of the largest forecast perturbation. Red perturbations are positive, blue are negative: (a) initial-time TE SV temperature perturbation; (b) 48-h evolved.

several possible sources for error in the computation of these AEC SVs. First, though the ensemble size was large, perhaps the singular vector structure would have been significantly different if the ensemble size were infinite. Second, we assume that errors grew linearly during the forecast. We would like to examine the validity of each of these assumptions.

On each of the case days, we have examined the structure of the leading AEC SV for ensembles of size 25,100 , and 400. It was clear that the structure of individual singular vectors was not fully converged with 400 members. However, of greater consequence for ensemble forecasting is the question of whether the leading singular vectors of small and larger ensembles span the same subspace. If they do, that subspace is converged, and adding additional ensemble members is not required. This aspect can be examined here by calculating how strongly the singular vectors of 25- or 100-member ensemble project onto the subspace of the leading SVs of the full 400-member ensemble, as done in Fig. 16. Such calculations were also demonstrated in Buizza (1994) and Hamill et al. (2000). The leading 25 initialtime singular vectors from the ensembles of size 25 and 100 projected moderately onto the subspace of the leading 25 singular vectors from the 400-member ensemble (Fig. 16a). The projection was much stronger for the evolved singular vectors (Fig. 16b), where for the first 10 singular vectors of the 100 -member ensemble, the projection was greater than $95 \%$. This suggests that there was noisiness in the initial-time AEC SVs that was somewhat irrelevant to the evolved structure, given a long-enough optimization time. This also indicates that a larger ensemble is required to represent analysis errors than to represent forecast errors (see also Hamill et al. 2002a). Barkmeijer et al. (1998, 1999) and others have previously noted that the evolved structure of ensembles tends to be very similar even when the initial ensembles are started from quite different structures. This similarity is a result of the attraction of all perturbations to the lower-dimensional strange attractor, which occurs on a timescale of 1-3 days (Snyder et al. 2003).

We examined the issue of linearity using the methodology outlined in Gilmour et al. (2001). They denote a nonlinearity index $\Theta$ as

$$
\Theta=\frac{\left\|\delta^{+}(t)+\delta^{-}(t)\right\|}{0.5\left\{\left\|\delta^{+}(t)\right\|+\left\|\delta^{-}(t)\right\|\right\}} .
$$

Here $\|\cdot\|$ denotes a total energy norm north of $20^{\circ} \mathrm{N}$, $\delta^{+}(t)$ denotes a positive perturbation, and $\delta^{-}(t)$ is a negative perturbation. When growth is linear, $\Theta=0$. To calculate $\Theta$ here, forecasts were integrated to $48 \mathrm{~h}$ from two perturbed initial conditions about the ensemble mean, one in the direction of the leading singular vector, the other its negative pair. Averaged over the 33 cases, 
$700 \mathrm{hPa}$ temperature and initial-time leading TE SV

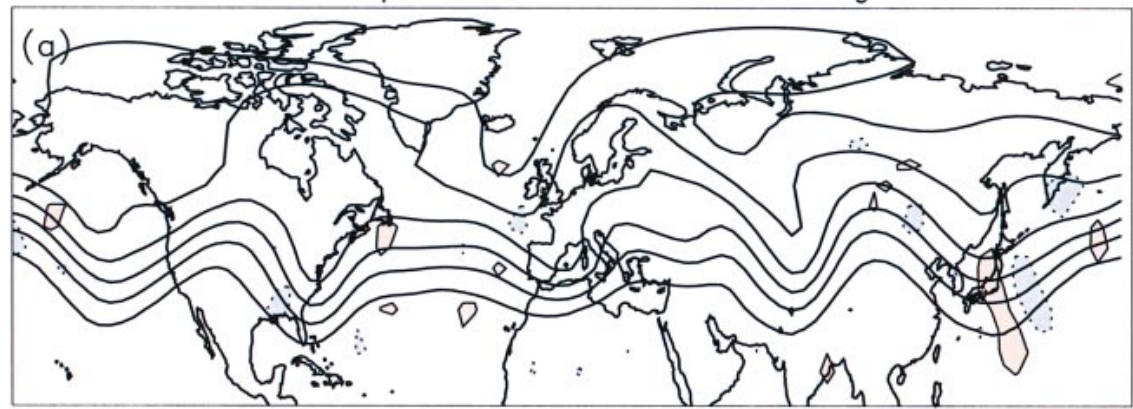

$700 \mathrm{hPa}$ temperature and evolved leading TE SV

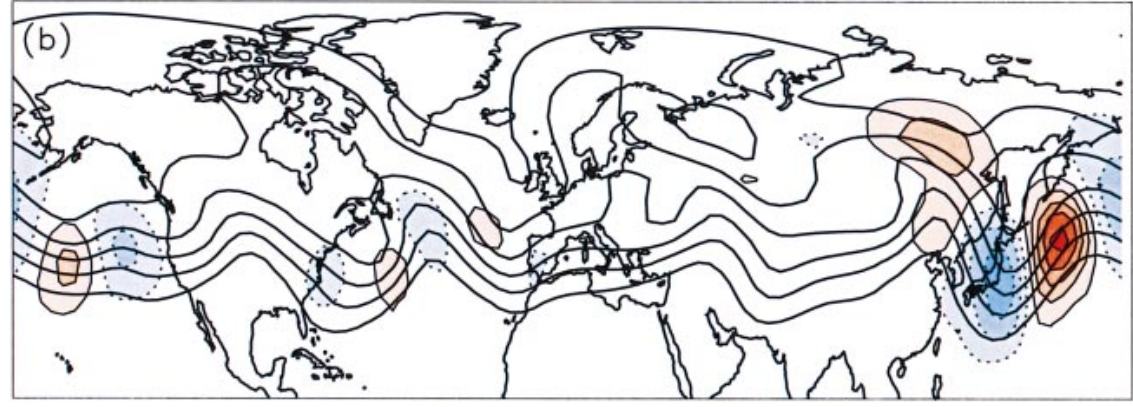

$300 \mathrm{hPa} \Phi$ and evolved leading TE SV

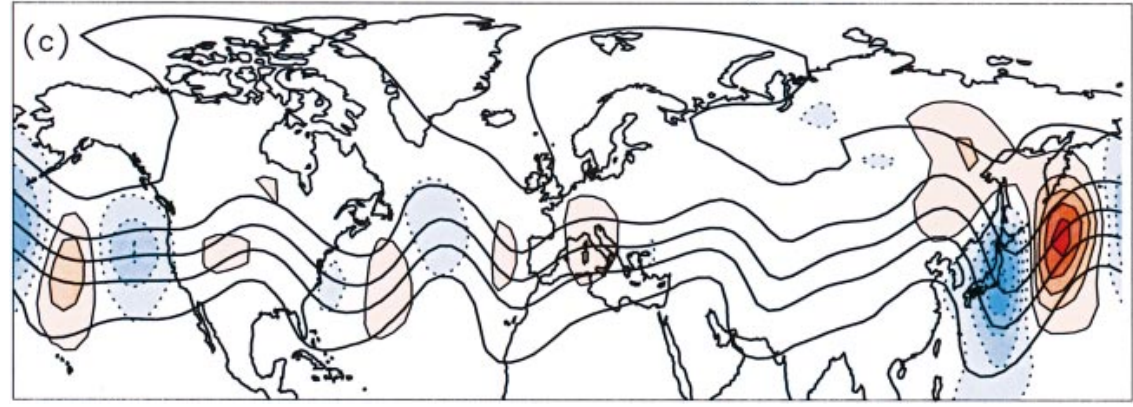

FIG. 11. Leading TE SV perturbations (colors) initialized on day 32.5: (a) initial-time 700-hPa temperature (solid lines, contours every $5 \mathrm{~K}$ ) and perturbations (normalized by the magnitude of the largest evolved perturbation); (b) 48-h 700-hPa temperature and evolved perturbations (same normalization); (c) 48-h 300-hPa streamfunction and evolved perturbations (normalized by the magnitude of the largest evolved streamfunction perturbation). Red perturbations are positive, blue are negative.

$\Theta$ was very small, typically $\sim 0.02$. Of course, as noted above, for a given model $\Theta$ will depend upon the size of the perturbation and the length of the forecast. Here, the nonlinearity was small both because the initial perturbations were very small and the timescale of the forecast was relatively short compared to the error-doubling time. These results are thus not generalizable to different forecast models with faster error growth rates and larger initial condition errors.

\section{Discussion and conclusions}

We have examined the structure of flow-dependent analysis-error covariance singular vectors (AEC SVs) from a simple general circulation model using an ensemble-based data assimilation system. This was done in order to understand the characteristics of singular vectors when their initial structure was constrained to be consistent with analysis error statistics. Further, this study demonstrates that the AEC SVs may be fundamentally different in structure than singular vectors using a total-energy initial norm. The structure of these singular vectors has important implications for how to generate dynamically constrained perturbations for ensemble forecasts.

In our experiments, a T31 L15 dry general circulation model (GCM) was used under perfect-model assumptions. Sets of 400-member ensembles of analyses were generated by an ensemble square root filter data assimilation system assimilating a sparse network of synthetic radiosonde observations. Ensembles of 48-h forecasts were also generated from these analyses. The evolved 

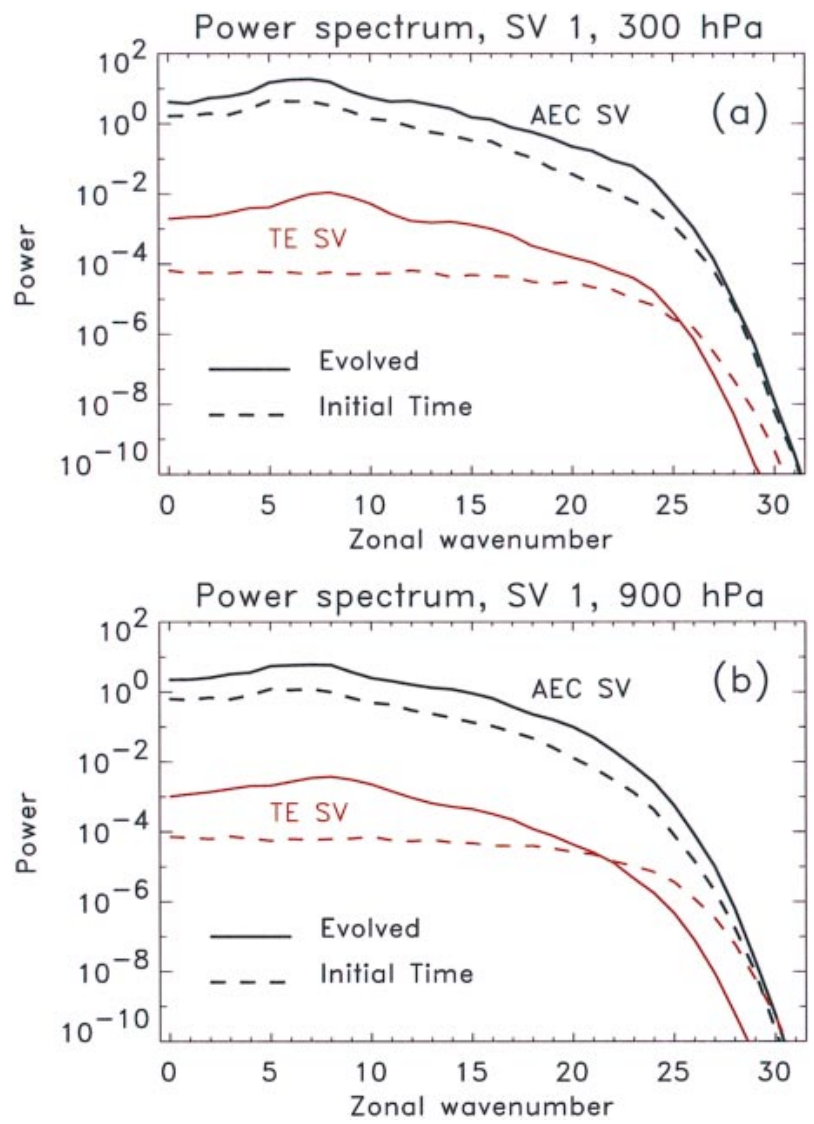

FIG. 12. Time- and latitudinally averaged power spectrum of zonal component of total energy for the leading singular vectors. Average is over 33 cases and latitudes from $35^{\circ}$ to $55^{\circ} \mathrm{N}$ latitude: power spectrum at (a) 300 and (b) $900 \mathrm{hPa}$.

singular vectors were determined by finding the linear combination of the forecast ensemble members that resulted in the largest forecast-error variance, here measured as a total-energy norm north of $20^{\circ} \mathrm{N}$ latitude. The same linear combination of analyses specified the initialtime structure that should evolve to the forecast singular vector under assumptions of linearity of error growth. A similar procedure was used to generate approximate total-energy singular vectors (TE SVs).

In this simplified GCM, the typical structure of initialtime TE SVs was significantly different than the structure of corresponding AEC SVs. The AEC SVs had maximum amplitude in midlatitudes near the tropopause, both at the initial and evolved times; the initial structure was very similar to the subsequent forecast error structure but smaller in magnitude. The AEC SVs were synoptic in scale and did not appear to be geographically localized. This contrasts with TE SVs, which started off much smaller in scale and had amplitudes that were typically largest in the lower to midtroposphere. The TE SVs grew very rapidly and changed in structure during the forecast, so that their evolved structure was rather similar to that of the evolved AEC SVs.

The structure of the AEC SVs shown here is generally consistent with the structure of Hessian singular vectors (HSVs) of Barkmeijer et al. (1998, 1999). For the HSVs, the initial norm is based on the Hessian of the cost function in a 3D-Var assimilation scheme; that Hessian matrix, in turn, is equal to the inverse of the analysiserror covariances that would be obtained if the background forecast error covariances assumed in 3D-Var were correct. Although the background-error covariances used in the HSVs are not flow dependent, the estimate of the analysis-error covariances provided by the Hessian is based on reasonable time-averaged estimates of the background-error covariances and a detailed accounting of the locations and accuracies of the available observations. Agreement of AEC SVs here with HSVs of Barkmeijer et al. further supports the relevance of our results to more complex and realistic NWP systems.

The structure of the AEC SVs is also consistent with estimates of analysis-error statistics in a quasigeostrophic model (Hamill et al. 2002a). In that model, the analysis errors share many of the characteristics of the AEC SVs calculated here: they are largest in the upper troposphere and are smallest in the lower to midtroposphere, they are synoptic in scale, and they are deep structures with weak vertical tilts. These properties of the analysis error in the quasigeostrophic model arise because of the rapid dynamical conditioning of errors during the forecast (Snyder et al. 2003) and because the analysis errors keep much of the character of the background errors, despite the effects of the assimilation process (Hamill et al. 2002a).

Perhaps with a more complex model than used in this experiment or with a change in experimental design (e.g., use of imperfect forecast models with moist thermodynamics) these inconsistencies of AEC SV and TE SV structures might change somewhat; this experiment used a low-resolution GCM under perfect-model assumptions. Nonetheless, there is clearly other evidence to support the relevance of our results. Conversely, there is little research to support the assertion that the structures of initial-time TE SVs are similar to those of AEC SVs.

What are the operational implications of this research? To the extent that analysis errors in our simplified model resemble those in global numerical weather prediction models, the difference in structure between these AEC SVs and ECMWF's TE SVs suggests that their operational ensemble forecasts could be improved from the change, were this computation practical. The choice of initial norm is likely to be especially important for short-range ensemble forecasts (SREFs). Because TE SVs grow more rapidly than AEC SVs, TE SV initial amplitudes are typically set below the magnitude of analysis error; this way, after their rapid initial growth they have a realistic magnitude (Molteni et al. 1996). 
$300 \mathrm{hPa} \Phi$ and initial time AEC SV 1

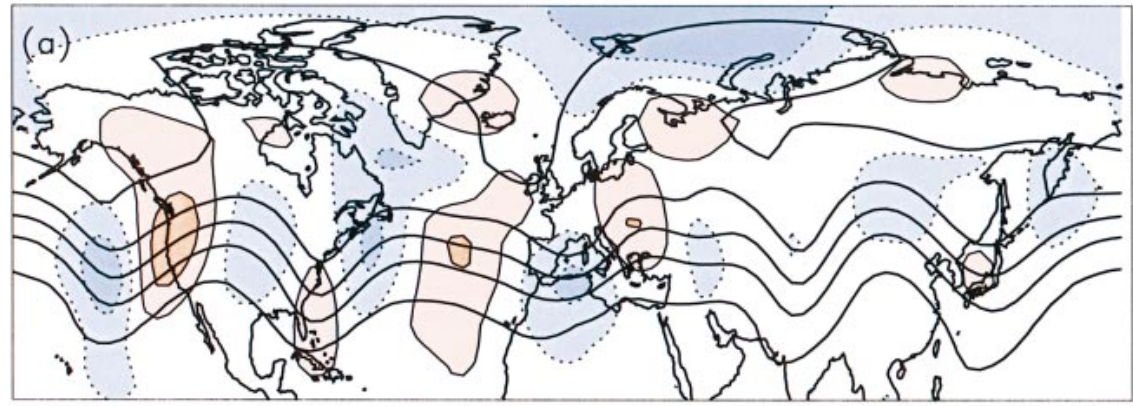

$300 \mathrm{hPa} \Phi$ and evolved AEC SV 1

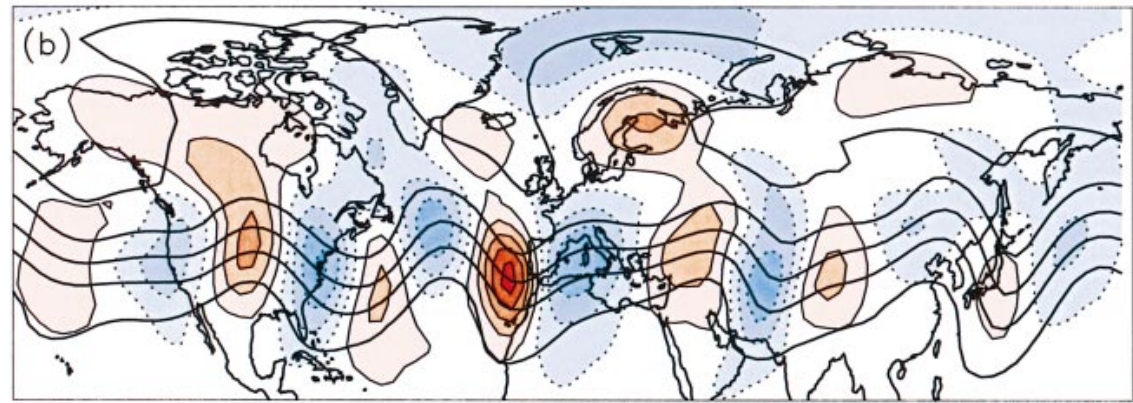

FIG. 13. The 300-hPa AEC SV structure for (a) initial time (here, day 32.5) and (b) 48-h evolved (here, day 34.5). Heavy solid lines denote streamfunction of true state at that time, and colored lines denote the perturbation to streamfunction from the first singular vector. Contours of true streamfunction are every $2 . \times 10^{7} \mathrm{~m}^{2} \mathrm{~s}^{-1}$. Streamfunction perturbations are normalized by the largest perturbation from the forecast, with contours at $[-0.9,-0.7, \ldots, 0.7,0.9]$. Red perturbations are positive, blue perturbations negative.

$300 \mathrm{hPa} \Phi$ and evolved AEC SV 1, 100 members

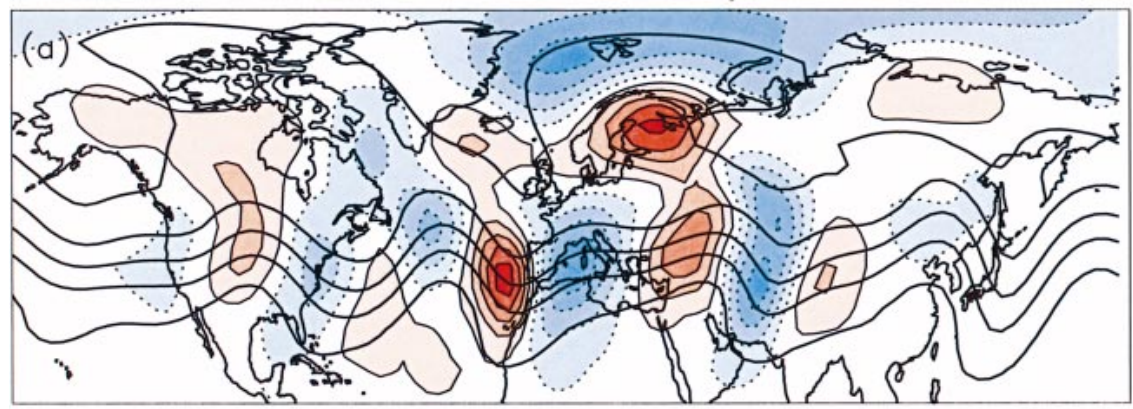

$300 \mathrm{hPa} \Phi$ and evolved AEC SV 1, 25 members

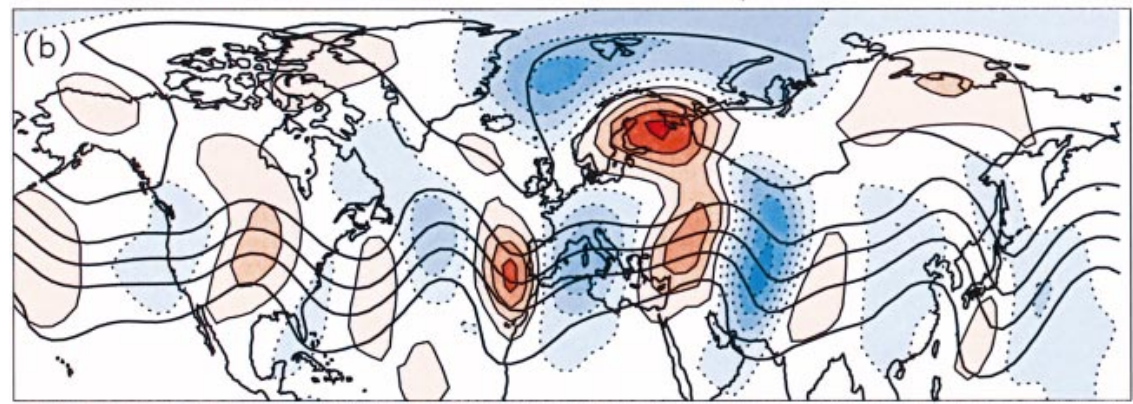

FIG. 14. (a) As in Fig. 13b, but from a 100-member ensemble; (b) as in Fig. 10b, but from a 25-member ensemble. 


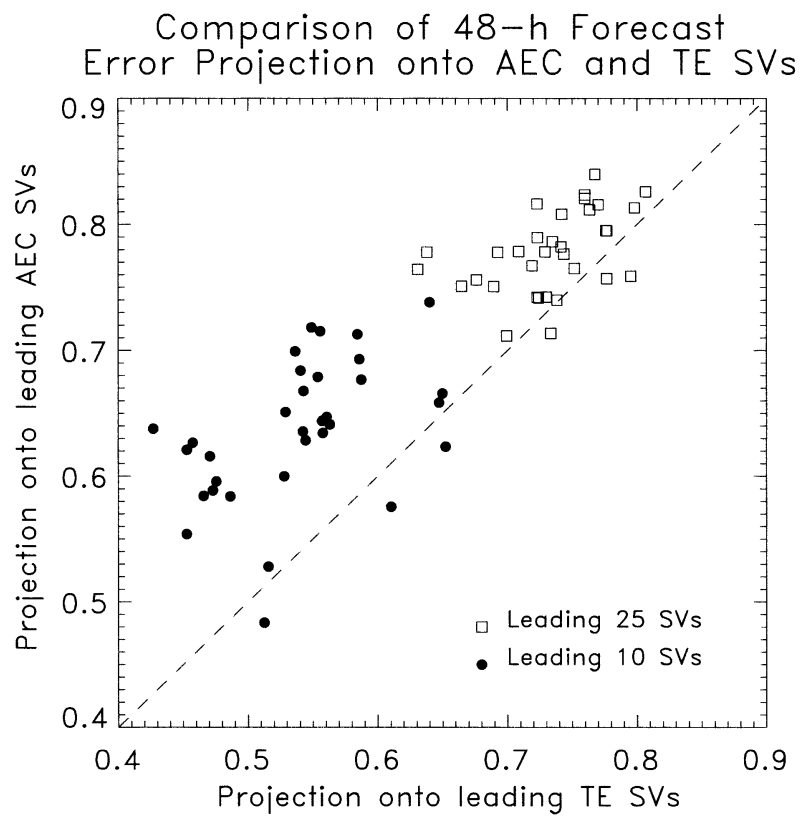

FIG. 15. Comparison of the projection of 48-h forecast error onto subspace of leading AEC SVs and TE SVs. Each dot denotes an individual forecast case (33 total). Squares denote the projection onto subspace of leading 25 singular vectors, filled circles onto the leading 10 singular vectors.

Further, their initial amplitudes are likely to be especially small near the surface and the tropopause, where the initial TE SV amplitude is typically small relative to AEC SV amplitude anyway. Consequently, SREFs from TE SV initial conditions can be expected to be unreliable at short lead times, having too little spread.

The calculation of AEC SVs in this study was facilitated by the existence of an ensemble-based data assimilation system. None of the major operational weather prediction facilities yet has such a data assimilation system in place, and the relative accuracy of ensemble data assimilation systems relative to 4D-Var is still unknown. Yet there are reasons for the operational centers to give serious consideration to these techniques. In this paper, we showed that analysis errors can be reduced to a remarkably low level with relatively few observations, albeit in a perfect-model simulation with a simple model. The key to this reduction was the flow-dependent background-error covariances generated by the ensemble filter, which produced a more optimal weighting of the background relative to the observations. The dramatically low errors demonstrated here do not seem to be unusual, either: a growing body of contemporaneous literature has also demonstrated the appeal of ensemble filters for atmospheric, oceanic, and land surface assimilation problems (Evensen 1994; Evensen and van Leeuwen 1996; Burgers et al. 1998; Houtekamer and Mitchell 1998, 2001; van Leeuwen 1999; Lermusiaux and Robinson 1999; Anderson and Anderson 1999; Hamill and Snyder 2000; Keppenne 2000; Mitchell and Houtekamer 2000; Heemink et al. 2001; Hamill et al.
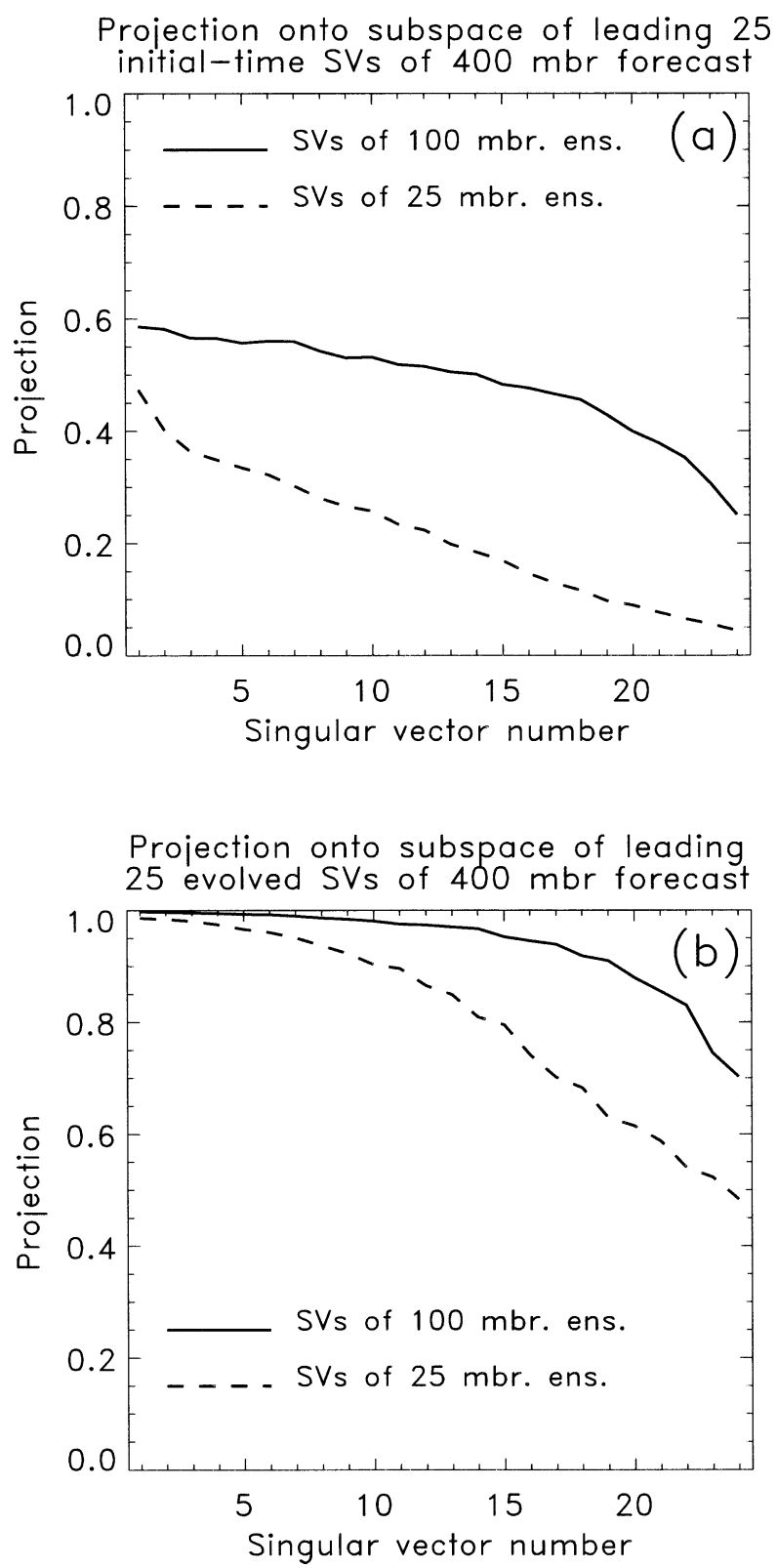

FIG. 16. Projection of leading AEC SVs from 25- and 100-member ensemble onto subspace of leading 25 AEC SVs from 400-member ensemble, averaged over all 33 cases: (a) for the initial-time AEC SVs; (b) for the evolved AEC SVs.

2001; Anderson 2001; Pham 2001; Whitaker and Hamill 2002; Reichle et al. 2002; Mitchell et al. 2002).

The appeal of ensemble filters may be greater yet if one considers the improvements they may foster for ancillary applications. Ensemble filters readily generate sets of initial conditions for ensemble forecasts that are automatically consistent with flow-dependent analysiserror statistics. They may facilitate more accurate computation of where adaptive observations are needed (Hamill and Snyder 2002), for parameter estimation 
(e.g., Anderson 2001), and for computation of AEC SVs, as demonstrated here.

An issue we have not addressed in this study is the relative value of ensemble forecasts initialized with random perturbations versus perturbations in the subspace of the singular vectors. The framework outlined in this paper provides a natural way of performing such tests, and we hope to address this in our future work.

Acknowledgments. The authors thank two anonymous reviewers for their careful reviews.

\section{REFERENCES}

Anderson, J. L., 1996: A method for producing and evaluating probabilistic forecasts from ensemble model integrations. J. Climate, 9, 1518-1530.

_ 1997: The impact of dynamical constraints on the selection of initial conditions for ensemble predictions: Low-order perfect model results. Mon. Wea. Rev., 125, 2969-2983.

__ 2001: An ensemble adjustment Kalman filter for data assimilation. Mon. Wea. Rev., 129, 2884-2903.

—_ and S. L. Anderson, 1999: A Monte Carlo implementation of the nonlinear filtering problem to produce ensemble assimilations and forecasts. Mon. Wea. Rev., 127, 2741-2758.

Badger, J., and B. J. Hoskins, 2001: Simple initial-value problems and mechanisms for baroclinic growth. J. Atmos. Sci., 58, 3849.

Barkmeijer, J., M. van Gijzen, and F. Bouttier, 1998: Singular vectors and estimates of the analysis error covariance metric. Quart. J. Roy. Meteor. Soc., 124, 1695-1713.

— R. Buizza, and T. N. Palmer, 1999: 3D-Var Hessian singular vectors and their potential use in the ECMWF ensemble prediction system. Quart. J. Roy. Meteor. Soc., 125, 2333-2351.

Bénard, P., A. Marki, P. N. Neytchev, and M. T. Prtenjak, 2000: Stabilization of nonlinear vertical diffusion schemes in the context of NWP models. Mon. Wea. Rev., 128, 1937-1948.

Boer, G. J., and T. G. Shepherd, 1983: Large-scale two-dimensional turbulence in the atmosphere. J. Atmos. Sci., 40, 164-184.

Buizza, R., 1994: Sensitivity of optimal unstable structures. Quart. J. Roy. Meteor. Soc., 120, 429-451.

— , and T. N. Palmer, 1995: The singular-vector structure of the atmospheric global circulation. J. Atmos. Sci., 52, 1434-1456.

_ J J. J. Tribbia, F. Molteni, and T. N. Palmer, 1993: Computation of optimal unstable structures for a numerical weather prediction model. Tellus, 45A, 388-407.

Burgers, G., P. J. van Leeuwen, and G. Evensen, 1998: Analysis scheme in the ensemble Kalman filter. Mon. Wea. Rev., 126, $1719-1724$.

Ehrendorfer, M., 1994: The Liouville equation and its potential usefulness for the prediction of forecast skill. Part I: Theory. Mon Wea. Rev., 122, 703-713.

_ , and R. M. Errico, 1995: Mesoscale predictability and the spectrum of optimal perturbations. J. Atmos. Sci., 52, 3475-3500.

— covariances through singular vectors. J. Atmos. Sci., 54, 286313.

Evensen, G., 1994: Sequential data assimilation with a nonlinear quasigeostrophic model using Monte Carlo methods to forecast error statistics. J. Geophys. Res., 99 (C5), 10 143-10 162.

_ , and P. J. van Leeuwen, 1996: Assimilation of Geosat altimeter data for the Agulhas current using the ensemble Kalman filter with a quasigeostrophic model. Mon. Wea. Rev., 124, 85-96.

Farrell, B. F., 1989: Optimal excitation of baroclinic waves. J. Atmos. Sci., 46, 1193-1206.

Gaspari, G., and S. E. Cohn, 1999: Construction of correlation func- tions in two and three dimensions. Quart. J. Roy. Meteor. Soc., 125, 723-757.

Gelaro, R., T. E. Rosmond, and R. Daley, 2002: Singular vector calculations with an analysis error variance metric. Mon. Wea. Rev., 130, 1166-1186.

Gilmour, I., L. A. Smith, and R. Buizza, 2001: Linear regime duration: Is 24 hours a long time in synoptic weather forecasting? J. Atmos. Sci. 58, 3525-3539.

Hamill, T. M., 2001: Interpretation of rank histograms for verifying ensemble forecasts. Mon. Wea. Rev., 129, 550-560.

—- and C. Snyder, 2000: A hybrid ensemble Kalman filter-3D variational analysis scheme. Mon. Wea. Rev., 128, 2905-2919.

__ and —, 2002: Using improved background error covariances from an ensemble Kalman filter for adaptive observations. Mon. Wea. Rev., 130, 1552-1572.

__ _ _ a and R. E. Morss, 2000: A comparison of probabilistic forecasts from bred, singular vector, and perturbed observation ensembles. Mon. Wea. Rev., 128, 1835-1851.

- J. S. Whitaker, and C. Snyder, 2001: Distance-dependent filtering of background error covariance estimates in an ensemble Kalman filter. Mon. Wea. Rev., 129, 2776-2790.

—_, — , and R. E. Morss, 2002a: Analysis-error statistics of a quasigeostrophic model using three-dimensional variational assimilation. Mon. Wea. Rev., 130, 2777-2790.

,$- \ldots$, and C. Snyder, 2002b: Generating initial conditions for ensemble forecasts: Monte-Carlo vs. dynamic methods. Preprints, Symp. on Observations, Data Assimilation, and Probabilistic Prediction, Orlando, FL, Amer. Meteor. Soc., J1-J6.

Heemink, A. W., M. Verlaan, and A. J. Segers, 2001: Variance-reduced ensemble Kalman filtering. Mon. Wea. Rev., 129, 1718 1728.

Held, I. M., and M. J. Suarez, 1994: A proposal for the intercomparison of the dynamical cores of atmospheric general circulation models. Bull. Amer. Meteor. Soc., 75, 1825-1830.

Hollingsworth, A., and P. Lönnberg, 1986: The statistical structure of short-range forecast errors as determined from radiosonde data. Part I: The wind field. Tellus, 38A, 111-136.

Hoskins, B. J., R. Buizza, and J. Badger, 2000: The nature of singular vector growth and structure. Quart. J. Roy. Meteor. Soc., 126, $1565-1580$.

Houtekamer, P. L., and J. Derome, 1995: Methods for ensemble prediction. Mon. Wea. Rev., 123, 2181-2196.

_ , and H. L. Mitchell, 1998: Data assimilation using an ensemble Kalman filter technique. Mon. Wea. Rev., 126, 796-811.

_ , and _ 2001: A sequential ensemble Kalman filter for atmospheric data assimilation. Mon. Wea. Rev., 129, 123-137.

_ _ L. Lafaivre, and J. Derome, 1996: The RPN ensemble prediction system. Proc. ECMWF Seminar on Predictability, Vol. II, Reading, United Kingdom, ECMWF, 121-146. [Available from ECMWF, Shinfield Park, Reading, Berkshire RG2 9AX, United Kingdom.]

Ide, K., P. Courtier, M. Ghil, and A. C. Lorenc, 1997: Unified notation for data assimilation: Operational, sequential, and variational. $J$. Meteor. Soc. Japan, 25, 181-189.

Keppenne, C. L., 2000: Data assimilation into a primitive equation model with a parallel ensemble Kalman filter. Mon. Wea. Rev. 128, 1971-1981.

Legras, B., and R. Vautard, 1996: A guide to Lyapunov vectors. Proc., ECMWF Seminar on Predictability, Vol. I, Reading, United Kingdom, ECMWF, 143-156. [Available from ECMWF, Shinfield Park, Reading, Berkshire RG2 9AX, United Kingdom.]

Lermusiaux, P. F. J., and A. R. Robinson, 1999: Data assimilation via error subspace statistical estimation. Part I: Theory and schemes. Mon. Wea. Rev., 127, 1385-1407.

Lorenc, A. C., 1986: Analysis methods for numerical weather prediction. Quart. J. Roy. Meteor. Soc., 112, 1177-1194.

Lorenz, E. N., 1963: Deterministic nonperiodic flow. J. Atmos. Sci., 20, 130-141.

Mitchell, H. L., and P. L. Houtekamer, 2000: An adaptive ensemble Kalman filter. Mon. Wea. Rev., 128, 416-433. 
and G. Pellerin, 2002: Ensemble size, balance, and model-error representation in an ensemble Kalman filter. Mon. Wea. Rev., 130, 2791-2808.

Molteni, F., R. Buizza, T. N. Palmer, and T. Petroliagis, 1996: The ECMWF ensemble prediction system: Methodology and validation. Quart. J. Roy. Meteor. Soc., 122, 73-119.

Morgan, M. C., 2001: A potential vorticity and wave activity diagnosis of optimal perturbation evolution. J. Atmos. Sci., 58, 2518 2544.

Mukogawa, H., and T. Ikeda, 1994: Optimal excitation of baroclinic waves in the Eady model. J. Meteor. Soc. Japan, 72, 499-513.

Parrish, D. F., and J. C. Derber, 1992: The National Meteorological Center's spectral statistical-interpolation analysis system. Mon. Wea. Rev., 120, 1747-1763.

Pham, D. T., 2001: Stochastic methods for sequential data assimilation in strongly nonlinear systems. Mon. Wea. Rev., 129, 1194-1207.

Reichle, R. H., D. B. McLaughlin, and D. Entekhabi, 2002: Hydro- logic data assimilation with the ensemble Kalman filter. Mon. Wea. Rev., 130, 103-114.

Reynolds, C. A., R. Gelaro, and J. D. Doyle, 2001: Relationship between singular vectors and transient features in the background flow. Quart. J. Roy. Meteor. Soc., 127, 1731-1760.

Snyder, C., T. M. Hamill, and S. J. Trier, 2003: Linear evolution of error covariances in a quasigeostrophic model. Mon. Wea. Rev., 131, 189-205.

Toth, Z., and E. Kalnay, 1993: Ensemble forecasting at NMC: The generation of perturbations. Bull. Amer. Meteor. Soc., 74, 2317 2330 .

_ , and _ 1997: Ensemble forecasting at NCEP and the breeding method. Mon. Wea. Rev., 125, 3297-3319.

van Leeuwen, P. J., 1999: Comment on "Data assimilation using an ensemble Kalman filter technique." Mon. Wea. Rev., 127, 13741377.

Whitaker, J. S., and T. M. Hamill, 2002: Ensemble data assimilation without perturbed observations. Mon. Wea. Rev., 130, 19131924. 